\title{
TRANSLATION-INVARIANT CONES OF FUNCTIONS ON SEMI-SIMPLE LIE GROUPS
}

\author{
HARRY FURSTENBERG ${ }^{1}$
}

Introduction. Originally, the phrase harmonic analysis had a function-theoretic meaning, referring to the decomposition of a function into exponentials. In the current interpretation, particularly in connection with noncommutative groups, the term refers not to functions but to representations, and harmonic analysis is regarded as part of the theory of group representations. This shift in interpretation was motivated by the $L^{2}$-theory for compact groups. The decomposition of the $L^{2}$-space of a noncommutative compact group analogous to the Fourier decomposition for the circle involves multidimensional subspaces, and, as a result, there is no longer a canonical choice of a basis for the $L^{2}$-space analogous to the set $\left\{e^{i n \theta}\right\}$ for the circle. The subspaces, on the other hand, are canonically determined, and correspond to the various irreducible representations of the group. It therefore became natural to regard irreducible representations as the basic building blocks of the theory in the place of the exponential functions.

Our purpose here is to call attention to some examples in the theory of semi-simple noncompact Lie groups where the classical setup prevails. That is, we shall find a class of functions on these groups which appear to play a role similar to that played by the exponentials for the circle or the real line. In terms of these functions, a form of spectral synthesis will be valid. Namely, for certain translationinvariant classes of functions on the group, we shall find that each function of such a class admits a unique representation as a generalized linear combination of the "exponentials" in that class. Admittedly, this result corresponds to a relatively easy case of spectral synthesis for the line. However, it is hoped that by pursuing this analogy further, other fruitful applications may be found.

The prototype of the theorem we shall prove is a theorem of Choquet and Deny [3] for $R^{n}$ (or any locally compact commutative group). Let $\mu$ be a positive bounded Borel measure on $R^{n}$ that does

An address delivered before the New York meeting of the Society on April 21, 1964 under the title Aspects of harmonic analysis on Lie groups, by invitation of the Committee to Select Hour Speakers for Eastern Sectional Meetings; received by the editors September 20, 1964.

${ }_{1}^{1}$ Work supported in part by Army Research Office, Project in Probability, Princeton University and the Air Force Office of Scientific Research. 
not have its support in a proper subgroup of $R^{n}$. Form the cone $V$ of (unbounded) positive measures $\nu$ on $R^{n}$ satisfying $\mu * \nu=\lambda \nu$, where $\lambda>0$ is fixed. A general result of Choquet and others to which we shall refer more explicitly later ensures that $V$ is spanned by its extremal rays. Moreover, choosing appropriately a single element from each extremal ray, there will be a unique integral representation of each member of $V$ in terms of these extremals. The question arises as to the nature of the extremal measures of $V$. The answer given by Choquet and Deny is that the extremal measures have the form $f(x) d x$ where $f(x)$ is proportional to an exponential: $f(x)=c e^{u \cdot x}$. In other words, the measures of $V$ all correspond to functions (in fact, convex functions), and these functions are linear combinations of the exponentials in $V: F \in V \Leftrightarrow F(x)=\int e^{u \cdot x} d \omega(u)$ where $\omega$ is a measure concentrated in the set of $u$ for which $\int e^{u \cdot x} d \mu(x)=\lambda$.

It is worthwhile sketching a proof of the fact that the extremals of $V$ correspond to exponentials. The cone $V$ is invariant under translation. Now the equation $\nu=\lambda^{-1} \mu * \nu$ implies that $\nu$ is a linear combination of its translates. This means that $\nu$ cannot be an extremal of $V$ unless all its translates by points in the support of $\mu$ are proportional to $\nu$. If the support of $\mu$ generates $R^{n}$ as a group, this implies that all translates of $\nu$ are proportional to $\nu$. One sees easily that $\nu$ must then be given by an exponential.

For a noncommutative group the convolution $\mu * \nu$ may still be defined and we can form the cone $V$ of solutions to $\mu * \nu=\lambda \nu$ as before. This time, however, $V$ is invariant under translation on the right: $\nu \rightarrow \nu * g$ (this will presently be defined more precisely) and the equation $\nu=\lambda^{-1} \mu * \nu$ implies that $\nu$ is a linear combination of left translates. The above argument, therefore, breaks down, and one can inquire what class of functions can occur as the extremals of $V$ in the general case. For a compact group $G$ this question is easily answered, because $V$ will be empty unless $\lambda=\mu(G)$ and $\nu$ must be proportional to the Haar measure of $G$. But for noncompact groups the question is more interesting and for the most part unsolved. We shall solve this problem only for semi-simple Lie groups, and even here only under additional hypotheses regarding $\mu$.

1. Preliminaries. If $G$ is a topological group, by a $G$-space is meant a space $X$ and a continuous map $(g, x) \rightarrow g x$ of $G \times X \rightarrow X$ satisfying $\left(g_{1} g_{2}\right) x=g_{1}\left(g_{2} x\right)$ and $e x=x$ for $e$ the identity of $G$. $X$ is a homogeneous $G$-space if $G$ is transitive on $X$; that is for $x, y \in X$, there exists $g \in G$ with $g x=y . X$ may then be identified with the coset space $G / H$, where, for some $x_{0} \in X, H$ is the subgroup that leaves $x_{0}$ fixed. 
If $X$ is a $G$-space, $\mu$ a Borel measure on $G$ and $\nu$ a Borel measure on $X$, then the convolution $\mu * \nu$ may be defined by

$$
\int_{X} f(y) d \mu * \nu(y)=\int_{X} \int_{G} f(g x) d \mu(g) d \nu(x),
$$

providing this integral is finite for continuous functions $f$ of compact support. In particular, the convolution of two bounded measures on $G$ is defined. We shall interchangeably denote the unit mass concentrated at a point $x$ of a space $X$ by $\delta_{x}$ or by $x$ itself. The convolution $\delta_{\theta} * \mu$ for $g \in G$ and $\mu$ a measure on a $G$-space (or on $G$ itself) will sometimes be denoted $g \mu$; similarly $\mu x$ is the same as $\mu * \delta_{x}$ where $\mu$ is a measure on $G$ and $x$ belongs to the $G$-space $X$. Notice that $\delta_{g} * \delta_{x}=\delta_{g x}$, so that our notation for convolution is consistent with the notation for multiplication. It is frequently useful to regard convolution as a linear extension of multiplication so that

$$
\mu * \nu=\int_{G} g \nu d \mu(g)=\int_{X} \mu x d \nu(x)=\int_{G} \int_{X} g x d \mu(g) d \nu(x) .
$$

The space of unbounded regular measures on a locally compact space may be identified with the dual of the space of continuous functions with compact support on the space. When we speak of the weak topology on measures, we have in mind the weak* topology of this dual space. In addition, it will often be convenient to denote an integral $\int f(x) d \mu(x)$ simply as $\mu(f)$.

On a locally compact group $G$ a locally integrable function $f(g)$ determines a measure $\omega$ on $G$ by $d \omega(g)=f(g) d g, d g$ denoting the left invariant Haar measure on $G$. We shall of ten find it convenient to identify the function $f$ with the measure $\omega$. Thus the convolution of two functions $f_{1} * f_{2}$, or of a function with a measure, $f * \mu$ or $\mu * f$, is defined as the function corresponding to the measure which is the convolution of the two measures involved.

On a group $G$ we use the notation $R_{\gamma}$ to denote the operator $R_{\gamma} f(g)$ $=f(g \gamma)$. It might be noticed that, as a measure, $R_{\gamma} f$ corresponds to the convolution $f * \delta_{\gamma^{-1}}$. In our discussion we shall be concerned primarily with translates on the right: $f \rightarrow R_{\gamma} f$. As a result we shall always understand, by the translate of a function $f$, a right translate $R_{\gamma} f$.

If $X$ and $Y$ are two $G$-spaces and $j$ is a map of $X$ into $Y$, we shall say that $j$ is equivariant if $j(g x)=g j(x)$, for all $x \in X, g \in G$.

By a semi-simple group $G$ we shall mean one which is noncompact and has a finite center. Such a group admits an Iwasawa decomposi- 
tion $G=K \cdot A \cdot N$. Here $K$ is any maximal compact subgroup of $G$, $A$ is a vector group and $N$ is nilpotent, $A \cdot N$ is a solvable group whose commutator subgroup is $N$. The decomposition is not direct but each $g \in G$ admits a unique representation $g=k a n$. The letter $K$ will always refer to a fixed but arbitrary maximal compact subgroup of $G$. $m_{K}$ denotes the normalized Haar measure on $K$. In general, if $\mu$ is a positive measure on a space $X$ and $\mu(X)=1$, we refer to $\mu$ as a probability measure.

2. Multipliers and multiplier functions. To generalize the notion of an exponential function to an arbitrary group we may proceed as follows. The exponentials are characterized by the property that they have unit value at the identity and any translate is proportional to the function itself. On a semi-simple group only the constant function has this property. (Such a function defines a homomorphism from the group to the complex numbers and the homomorphic image of a semi-simple group is semi-simple.) So we modify this condition by supposing instead that we have a family of functions $s(\cdot, \xi)$, where $\xi$ ranges over an index set $X$, with the property that $s(e, \xi)=1$ and that a (right) translate of each of the $s(\cdot, \xi)$ is proportional to some other of the $s(\cdot, \xi)$. We may take $X$ to be the family of functions occurring here with the identification of proportional functions. $M$ is then a $G$-space, and we may write $R_{\gamma} s(\cdot, \xi) \sim s(\cdot, \gamma \xi)$. More precisely, we have

$$
R_{\gamma} s(g, \xi)=s(g \gamma, \xi)=h_{\xi}(\gamma) s(g, \gamma \xi) .
$$

Setting $g=e$, we have $s(\gamma, \xi)=h_{\xi}(\gamma)$ or

$$
s\left(g_{\gamma}, \xi\right)=s(g, \gamma \xi) s(\gamma, \xi) .
$$

A function satisfying (2.1) is usually referred to as a multiplier. As a function from $G$ to the module of functions on $M$ it is referred to as a crossed homomorphism or a 1-cocycle. Our usage will be more restrictive and we take the following as our definition.

Definition 2.1. Let $X$ be a $G$-space. A continuous function $s$ from $G \times X$ to the positive reals is called a multiplier if it satisfies (2.1). The group of all such functions is denoted $M(X)$ (where the group $G$ is assumed fixed). If $s \in M(X)$ and $\xi \in X$ then the function $s(\cdot, \xi)$ is called a multiplier function. The set of all multiplier functions corresponding to multipliers in $M(X)$ is denoted $E(X)$.

If $X$ consists of a single point, then (2.1) defines a positive character (homomorphism into $(0, \infty)$ ) on $G$. Thus the positive exponen- 
tials are the multiplier functions on $R^{n}$ corresponding to a trivial $X$. As we have remarked, for $G$ semi-simple, if $X$ is trivial, then $E(X)$ consists of the function 1. On the other hand, if $X$ is taken too large, $E(X)$ is again uninteresting because it contains all positive continuous functions $f$ with $f(e)=1$. For example, if we take $X=G$, and if $f$ satisfies $f(e)=1$, define $s \in M(G)$ by $s\left(g_{1}, g_{2}\right)=f\left(g_{1} g_{2}\right) / f\left(g_{2}\right)$. It is easily seen that $s$ satisfies $(2.1)$ and $f(g)=s(g, e)$. What we shall find is that in the case of a semi-simple $G$, there is a choice of $X$ for which the functions of $E(X)$ play the role of the exponentials. In particular, these functions will turn out to be the extremals in our version of the Choquet-Deny theorem.

If $p(\xi)$ is a positive continuous functions on the $G$-space $X$, we may form a multiplier in $M(X)$ by setting

$$
s(g, \xi)=p(g \xi) / p(\xi) .
$$

We call these multipliers trivial. (In the terminology of cohomology theory, these would be coboundaries.) We shall call two multipliers equivalent if their quotient is trivial. The (cohomology) group of equivalence classes will be denoted $H(X)$.

Similar considerations arise in the representation theory of $G$. If $X$ is a $G$-space we may define a representation of $G$ on some linear space of functions on $X$ by setting $U_{\theta} f(\xi)=s\left(g^{-1}, \xi\right) f\left(g^{-1} \xi\right)$. The condition that $U_{o}$ be a representation is just the multiplier equation (2.1) for $s$. Moreover, if $U_{\theta}$ and $V_{\theta}$ arise from equivalent multipliers, then $U_{o}$ and $V_{o}$ are equivalent representations. It will not be surprising that the space $X$ that we will study has also been studied in connection with the irreducible representations for semi-simple Lie groups [1].

Let $G$ be semi-simple and $K$ a maximal compact subgroup, and let $X$ be a $G$-space.

Definition 2.2. $\sigma$ is a $K$-multiplier if $\sigma \in M(X)$ and $\sigma(k, \xi)=1$ for all $k \in K, \xi \in X$. The set of $K$-multipliers forms a group $M_{K}(X)$, and the corresponding set of multiplier functions is denoted $E_{K}(X)$.

Lemma 2.1. Let $X$ be a homogeneous $G$-space and suppose that the subgroup $K$ is already transitive on $X$. Then the natural map of $M_{K}(X)$ into $H(X)$ is an isomorphism onto.

Proof. To show that this map is 1-1 suppose $\sigma \in M_{K}(X)$ and is trivial: $\sigma(g \xi),=p(g \xi) / p(\xi)$. Then $p(k \xi)=p(\xi)$ and since $K$ is transitive, $p$ is constant so that $\sigma \equiv 1$. To show the map is onto we associate to every multiplier an equivalent $K$-multiplier. For $s \in M(X)$, set 


$$
\sigma(g, \xi)=\frac{\int_{K} s(k g, \xi) d k}{\int_{K} s(k, \xi) d k}=\frac{\int_{K} s(k, g \xi) d k}{\int_{K} s(k, \xi) d k} s(g, \xi),
$$

from which we see that $\sigma \sim s$ and $\sigma(k, \xi)=1$.

From this we can see that, in many cases, although the group $M(X)$ is infinite-dimensional, the group $H(X)$ is finite-dimensional. For if we let $X=G / L$, where $L$ is a subgroup of $G$, then $K$ is transitive on $X$ if and only if $K L=G$. If $\sigma \in M_{K}$ and $\xi_{0} \in G / L$ corresponds to the coset $L$, then $\sigma(g, \xi)=\sigma\left(g, k \xi_{0}\right)=\sigma\left(g k, \xi_{0}\right)$ for some $k \in K$. So $\sigma$ is determined by the function $\sigma\left(g, \xi_{0}\right)$. Since $g=k^{\prime} l$ for some $k^{\prime} \in K, l \in L, \sigma$ is determined by the restriction of $\sigma\left(\cdot, \xi_{0}\right)$ to $L$. But this is a positive character by (2.1), since $l \xi_{0}=\xi_{0}$. In particular, if $L$ has finitely many connected components then its (positive) character group is a subgroup of a vector group. It follows that $M_{K}(X)$ and, by Lemma 2.1, also $H(X)$, is isomorphic to a subgroup of a vector group.

In certain cases we can assert that $H(X)$ is trivial.

Lemma 2.2. If $L$ is compact then $H(G / L)=1$.

Proof. Let $s \in M(G / L)$ and let $\xi_{0} \in G / L$ correspond to the coset $L$. Then $s\left(l, \xi_{0}\right)$ is a positive character on $L$. But, since $L$ is compact, $s\left(l, \xi_{0}\right)=1$. It follows that $s\left(g l, \xi_{0}\right)=s\left(g, l \xi_{0}\right) s\left(l, \xi_{0}\right)=s\left(g, \xi_{0}\right)$, so that $s\left(g, \xi_{0}\right)=p\left(g \xi_{0}\right)$ for some positive continuous function $p$ on $G / L$. Hence $s\left(g, g^{\prime} \xi_{0}\right)=s\left(g g^{\prime}, \xi_{0}\right) / s\left(g^{\prime}, \xi_{0}\right)=p\left(g g^{\prime} \xi_{0}\right) / p\left(g^{\prime} \xi_{0}\right)$ or $s(g, \xi)=p(g \xi) / p(\xi)$.

As a final remark we point out that if $j$ is an equivariant map of one $G$-space $X$ into another $G$-space $Y$, then there is induced a map $M(Y) \rightarrow M(X)$. Namely we assign to each $s \in M(Y)$ the multiplier $s^{*}$ defined by $s^{*}(g, x)=s(g, j(x)) . s^{*}$ satisfies (2.1) if $s$ does. If $K$ is transitive on $X$, then it is possible to show that the map of $M(Y)$ $\rightarrow M(X)$ induces an isomorphism of $H(Y)$ into $H(X)$.

3. Examples. In this section we shall recall some familiar integral representation formulae which we shall see express a function as a linear combination of multiplier functions. In each case the function being represented satisfies a convolution equation or a family of such equations, and the formula will turn out to be an instance of the generalized Choquet-Deny representation.

Let $G$ be the connected group of rigid motions of the plane $R^{2}$. $G$ is the semidirect product $K \cdot R^{2}$ where $K$ is the subgroup of notations and $R^{2}$ the subgroup of pure translations. $R^{2}$ is a normal subgroup of $G$ and $G / R^{2}$ may be identified with $K$. We shall compute $M_{K}\left(G / R^{2}\right)$. 
$1965]$

TRANSLATION-INVARIANT CONES OF FUNCTIONS

277

(Note that by Lemma 2.2, $H(G / K)$ is trivial, so that all multipliers of $M(G / K)$ are trivial.) Identifying $\boldsymbol{R}^{2}$ with the complex plane, we may denote an element $g \in G$ by the pair $\left(e^{i \theta}, w\right)$ if $g(z)=e^{i \theta} z+w$. The action of $G$ on $K=G / R^{2}$ is given by $\left(e^{i \theta}, w\right) \zeta=e^{i \theta} \zeta$ where $|\zeta|=1$. We then find that if $\sigma \in M_{K}\left(G / R^{2}\right)$,

$$
\sigma\left(e^{i \theta}, w ; \zeta\right)=\sigma\left(1, e^{-i \theta \zeta} \zeta^{-1} w ; 1\right)=e^{\Delta\left(e^{-i \theta} \zeta^{-1} w\right)},
$$

where $\Lambda$ denotes a homomorphism of $C \rightarrow R$. This means $\Lambda(z)=R z \tau$ for some complex $\tau$. The general form of a multiplier in $M_{K}\left(G / R^{2}\right)$ is therefore

$$
\sigma_{\tau}\left(e^{i \theta}, w ; \zeta\right)=e^{\mathcal{R e}^{-i \theta_{\zeta}-1} w \tau}
$$

Consider now the zero-order Bessel function of an imaginary argumint: $f(r)=J_{0}(i r)$. The function on $R^{2}$ given by $F(z)=f(|z|)$ is an eigenfunction of the Laplacian $\Delta=D_{x}^{2}+D_{y}^{2}$ on the plane. This implies that it is an eigenfunction of the mean-value operators:

$$
T_{\rho} F(z)=\frac{1}{2 \pi} \int_{0}^{2 \pi} F\left(z+\rho e^{i \theta}\right) d \theta .
$$

We now lift the function $F(z)$ to the group $G$ by setting $\Phi\left(e^{i \theta}, w\right)$ $=F(w)$. If we write the equations $T_{\rho} F=\lambda_{\rho} F$ in terms of $\Phi$ we find that $\Phi$ satisfies a family of convolution equations on the group. According to the theorem to be proven it follows from this that $\Phi$ can be expressed as an integral over a set of multiplier functions in $E\left(G / R^{2}\right)$. Moreover, since $\Phi(k g)=\Phi(g)$ for $k \in K$, these multiplier functions will be in $E_{K}\left(G / R^{2}\right)$, and so are given by (3.1). This is indeed the case, for by the Bessel formula

$$
f(r)=\frac{1}{2 \pi} \int_{0}^{2 \pi} e^{-r \cos \omega} d \omega=\int e^{-Q_{r 5^{-1}}} d m(\zeta),
$$

where $d m(\zeta)$ refers to normalized Lebesgue measure on the unit cirche. It follows that

$$
\begin{aligned}
F(z) & =\int e^{-R_{z \zeta}} d m(\zeta), \\
\Phi\left(e^{i \theta}, w\right) & =\int e^{-\Re_{w} \zeta^{-1}} d m(\zeta)=\int e^{-R_{w e^{-i \theta \zeta^{-1}}} d m(\zeta)} \\
& =\int \sigma_{1}\left(e^{i \theta}, w ; \zeta\right) d m(\zeta) .
\end{aligned}
$$


Bessel's formula is a special case of a formula for spherical functions on any symmetric space of noncompact type. (See [8, Chapter X] for details.) Let us consider the case of a space of constant negative curvature. The space may then be taken as $G / K$ where $G$ is semisimple. A spherical function $F(x)$ on $G / K$ is defined by the conditions: $F(k x)=F(x)$ for all $k \in K, F\left(x_{0}\right)=1$ where $x_{0}$ corresponds to the coset $K$, and $F$ is an eigenfunction of all differential operators on $G / K$ which commute with the action of $G$ on $G / K$. If $F(x)$ is lifted to $G$ we obtain a function $\phi(g)=F(g K)$ which, as may be shown, is characterized by the equation:

$$
\int_{K} \phi\left(g_{1} k g_{2}\right) d k=\phi\left(g_{1}\right) \phi\left(g_{2}\right), \quad g_{1}, g_{2} \in G .
$$

We notice that $\phi$ satisfies a family of convolution equations. In fact, (3.2) may be rewritten (see §1) as

$$
m_{K} * g_{1}^{-1} * \phi=\phi\left(g_{1}\right) \phi .
$$

The integral representation formula for these functions is due to Harish-Chandra [8, Chapter X, Theorem 6.16]. We define a function $H$ from $G$ to $A$, where $A$ is the vector part of the Iwasawa decomposition of $G$, by $H(k a n)=a$. Then, for every spherical function $\phi$ on $G$, there is a homomorphism $\Lambda: A \rightarrow C$ with

$$
\phi(g)=\int_{K} e^{\Lambda(B(o k))} d k .
$$

When $\phi$ is real and positive, $\Lambda$ is real-valued. We shall show that in this case, the integrand of (3.4) represents a multiplier in $M_{K}(G / A N)$, where $K A N$ is the Iwasawa decomposition of $G$. Namely, set $\sigma(g, k A N)$ $=e^{\Delta(H(o k))}$. Since $H\left(k^{\prime}\right)$ is the identity of $A$ if $k^{\prime} \in K$, it follows that $\sigma\left(k^{\prime}, k A N\right)=1$. We next check the multiplier equation. Suppose $\xi=k A N$ for $k \in K$, and $g_{1}, g_{2} \in G$ with

$$
g_{2} k=k_{2} a_{2} n_{2}, \quad g_{1} k_{2}=k_{1} a_{1} n_{1} .
$$

Then $g_{1} g_{2} k=g_{1} k_{2} a_{2} n_{2}=k_{1} a_{1} n_{1} a_{2} n_{2}=k_{1} a_{1} a_{2} n_{1}^{\prime} n_{2}$ since $N$ is normal in $A N$. Then

$$
\begin{aligned}
\sigma\left(g_{1} g_{2}, \xi\right) & =e^{\Lambda\left(H\left(o_{1} g_{2} k\right)\right)}=e^{\Lambda\left(a_{1}\right)+\Delta\left(a_{2}\right)}, \\
\sigma\left(g_{1}, g_{2} \xi\right) & =\sigma\left(g_{1}, k_{2} A N\right)=e^{\Lambda\left(H\left(o_{1} k_{2}\right)\right)}=e^{\Lambda\left(a_{1}\right)}, \\
\sigma\left(g_{2}, \xi\right) & =\sigma\left(g_{2}, k A N\right)=e^{\Lambda\left(H\left(o_{2} k\right)\right)}=e^{\Lambda\left(a_{2}\right)},
\end{aligned}
$$

so that $\sigma\left(g_{1} g_{2}, \xi\right)=\sigma\left(g_{1}, g_{2} \xi\right) \sigma\left(g_{2}, \xi\right)$. (3.4) may therefore be rewritten 


$$
\phi(g)=\int_{K} \sigma(g, k A N) d k=\int_{G / A N} \sigma(g, \xi) d m(\xi) .
$$

Thus the Harish-Chandra formula in the case of positive spherical functions is a special case of the generalized Choquet-Deny representation. It should be pointed out that our methods fail in the case of complex-valued spherical functions although the formula (3.4) is still valid.

Another example of a spectral decomposition for functions on a semi-simple group is given by the Poisson formula for harmonic functions in the unit disc. Let $G$ denote the connected group of conformal maps of the unit disc $D=\{z:|z|<1\}$ onto itself. A function $h(z)$ on the disc determines a function $f(g)=h\left(g^{-1}(0)\right)$ on $G$. Let $K$ again denote the group of rotations about the origin. The mean-value property for harmonic functions says that $h(0)=\int_{K} h(k z) d k$ for any $z \in D$. If $h$ is harmonic, so is the function obtained by composing $h$ with the transformation $g^{-1}, g \in G$. This gives

$$
h\left(g^{-1}(0)\right)=\int_{\boldsymbol{K}} h\left(g^{-1} k z\right) d k .
$$

If we write $z=\gamma^{-1}(0)$, (3.6) becomes

$$
f(g)=\int_{K} f(\gamma k g) d k, \quad g, \gamma \in G .
$$

Thus the functions $f(g)$ which correspond to harmonic functions on $D$ satisfy a family of convolution equations:

$$
m_{K} * \gamma^{-1} * f=f .
$$

Assume that $h \geqq 0$. The Poisson formula gives

$$
h(z)=\int_{0}^{2 \pi} P(z, \theta) d \nu(\theta),
$$

where $P$ denotes the Poisson kernel, and $\nu$ is a positive measure. Now one may verify that if $z=g^{-1}(0)$ then

or

$$
P(z, \theta)=\left|g^{\prime}\left(e^{i \theta}\right)\right|
$$

$$
f(g)=\int_{0}^{2 \pi}\left|g^{\prime}\left(e^{i \theta}\right)\right| d \nu(\theta)
$$


We recognize by the formula for differentiating a composite function that the integrand in (3.9) is a multiplier in $M_{K}(\partial D)$ where $\partial D$ is the unit circle.

The Poisson formula for harmonic functions also generalizes to arbitrary noncompact symmetric spaces. Moreover, a similar representation is valid for any positive eigenfunction of the Laplace-Beltrami operator on a symmetric space. This was shown by Dynkin [5] for the symmetric space $\mathrm{SL}(n, C) / \mathrm{SU}(n)$. Once again it may be shown that the extremal solutions occurring in Dynkin's representation are multiplier functions for a certain compact $G$-space which we will call $B(G)$ and regarding which we shall presently be more specific.

Another generalization of the Poisson formula for harmonic functions was given in [6]. For our present purposes it will be convenient to deviate somewhat from the notation used in [6]. Let $\mu$ be a probability measure on $G$ which is absolutely continuous and whose support contains a neighborhood of the identity of $G$. Consider the bounded solutions to

$$
f(g)=\int_{G} f\left(g^{\prime} g\right) d \mu\left(g^{\prime}\right) .
$$

This is a generalization of (3.7) which was satisfied by ordinary harmonic functions. It is shown that, given $\mu$, there is determined a probability measure $\nu$ on the $G$-space $B(G)$ referred to in the preceding paragraph, with the property that every bounded solution of (3.10) is given by

$$
f(g)=\int_{B(G)} f\left(g^{-1} x\right) d \nu(x),
$$

where $f$ is a bounded measurable function on $B(G)$. The measure $\nu$ satisfies $\mu * \nu=\nu$ and from this one can infer that the transforms $g \nu$ are absolutely continuous with respect to $\nu$, and that $(d g \nu / d \nu)(x)$ is positive and continuous on $G \times B(G)$. (3.11) may therefore be written

$$
f(g)=\int_{B(G)} \frac{d g^{-1} \nu}{d \nu}(x) f(x) d \nu(x) .
$$

We claim that $s(g, x)=\left(d g^{-1} \nu / d \nu\right)(x)$ is a multiplier in $M(B(G))$. By the definition of the Radon-Nikodym derivative it is easily seen that

$$
\frac{d g^{-1} \nu_{1}}{d g^{-1} \nu_{2}}(x)=\frac{d \nu_{1}}{d \nu_{2}}(g x)
$$




$$
\begin{aligned}
\frac{d g_{2}^{-1} g_{1}^{-1} \nu}{d \nu}(x) & =\frac{d g_{2}^{-1} g_{1}^{-1} \nu}{d g_{2}^{-1} \nu}(x) \frac{d g_{2}^{-1} \nu}{d \nu}(x) \\
& =\frac{d g_{1}^{-1} \nu}{d \nu}\left(g_{2} x\right) \frac{d g_{2}^{-1} \nu}{d \nu}(x),
\end{aligned}
$$

which is the multiplier equation for $s$. Thus the measure $\mu$ on $G$ determines a multiplier $s \in M(B(G))$ and the bounded solutions of (3.10) are integrals over multiplier functions corresponding to $s$ :

$$
f(g)=\int_{B(G)} s(g, x) f(x) d \nu(x)
$$

We note that this is the first example in which a multiplier is used which is not a $K$-multiplier. Naturally, a function cannot be represented in terms of $K$-multiplier functions unless it satisfies $f(k g)$ $=f(g)$ for $k \in K$.

It should be mentioned that in case the support of $\mu$ does not contain a neighborhood of the identity a similar representation is valid, but the relevant space may not be $B(G)$ but rather one of finitely many covering spaces of $B(G)$.

The space $B(G)$ which occurs in these formulas may be defined as follows. If $G=K A N$ is an Iwasawa decomposition of $G$ we let $T$ denote the normalizer of $A N$, that is the set of all $g \in G$ with $g A N g^{-1}$ $C A N . T$ is also obtained by adjoining to $A N$ the centralizer $K_{0}$ of $A$ in $K: T=K_{0} A N . B(G)$ is then defined as $G / T$. T is not uniquely determined, but it is unique up to conjugacy. As a result, the various versions of $G / T$ are all isomorphic as $G$-spaces. An alternative definition of $B(G)$ occurs in [6]. We define a boundary of a Lie group $G$ to be a compact $G$-space with the property that for any probability measure $\pi$ on the space, some sequence $g_{n} \pi, g_{n} \in G$, converges to a point measure. All boundaries of $G$ are equivariant images of one of them, and this one is $B(G)$. The equivalence of the two definitions was proven in [10].

The space $B(G)$ is an equivariant image of the $G$-space $G / A N$ that occurs in (3.5). It may be shown that, in fact, the multiplier $\sigma$ in formula (3.5) which belongs to $M_{K}(G / A N)$ actually is the image of a multiplier in $M_{K}(B(G)$ ) (see end of $\$ 2$ ) so that the integral in (3.5) may also be taken over $B(G)$. Thus in each of the cases studied, the integral representation involves multipliers in $M(B(G))$.

4. Tychonoff groups. The significance of the subgroup $T$ may be attributed to the fact that it possesses a certain "fixed-point" property which we will elucidate here. We consider a subcone $V$ of a linear 
topological space $S$. We say that $V$ has a compact base if there exists a continuous linear functional $L$ on $S$ such that $L(x)>0$ for $x \in V$ except for $x=0$, and such that $V_{1}=\{x \in V: L(x)=1\}$ is compact. If $\tau$ is a continuous linear transformation of $S$ taking $V$ into itself then $\tau$ has a fixed (invariant) ray in $V$. For, by the Schauder-Tychonoff fixed-point theorem [4], the transformation

$$
x \rightarrow \frac{\tau x}{L(\tau x)}
$$

which is continuous and takes $V_{1} \rightarrow V_{1}$ must have a fixed point in $V_{1}$. The ray through this fixed point is clearly left fixed by $\tau$. This result may be extended to a one-parameter group of transformations $\tau(t)$ of $S$ which take $V$ into itself, and there will exist a ray in $V$ invariant under the entire group.

Definition 4.1. A Lie group $G$ is a Tychonoff group if, whenever $G$ acts continuously by linear transformations on a locally convex, linear, topological space $S$, taking a cone $V$ with compact base into itself, then $G$ has a fixed ray in $V$.

A Tychonoff group also has the following fixed-point property: If it acts by affine transformations on a compact convex set, it has a fixed point in the set. (An affine transformation is one that preserves the convex structure: $\tau(\alpha x+(1-\alpha) y)=\alpha \tau x+(1-\alpha) \tau y, 0 \leqq \alpha \leqq 1$.) The converse, however, is not true; a group may have the latter fixedpoint property without being a Tychonoff group. For example, every solvable group has the fixed-point property relative to compact convex sets. (See [6].) On the other hand, the group of matrices

$$
\left\{\left(\begin{array}{ll}
x & 0 \\
0 & y
\end{array}\right),\left(\begin{array}{ll}
0 & u \\
v & 0
\end{array}\right): x, y, u, v>0\right\}
$$

is solvable, maps the first quadrant of $\boldsymbol{R}^{2}$ into itself, but has no fixed ray. An example of a connected solvable group which is not a Tychonoff group is the connected group of rigid motions of the plane. It will develop that if this group were a Tychonoff group, there would be no nonconstant positive eigenfunctions of the Laplacian in the plane.

The following lemmas will be useful in establishing that certain groups are Tychonoff groups.

LeMma 4.1. Let $A$ be a linear transformation on $R^{r}$ with strictly positive eigenvalues. If for some vector $x$, the set of vectors $\left\{A^{n} x:-\infty<n<\infty\right\}$ is bounded, then $A x=x$. 
Proof. Since $A$ satisfies its characteristic equation, it follows that all the vectors $A^{n} x$ are contained in the subspace $H$ spanned by $\left\{x, A x, \cdots, A^{r-1} x\right\}$. Clearly each vector $y \in H$ has the property that the set $\left\{A^{n} y\right\}$ is bounded. So the restriction of $A$ to $H$ is an operator with all its powers, positive and negative, bounded. Therefore all its eigenvalues are on the unit circle. But they must be positive and so the restriction of $A$ to $H$ has only the eigenvalue 1. We may therefore express this restriction as $I+N$, where $I$ is the identity on $H$ and $N$ is nilpotent on $H$. Let $i$ be the least integer with $N^{i}=0$. If $i \geqq 2$, form $(I+N)^{n} N^{i-2}=N^{i-2}+n N^{i-1}$. This must be bounded as $n \rightarrow \infty$ which implies $N^{i-1}=0$ and this is a contradiction. It follows that $N=0$, or the restriction of $A$ to $H$ is the identity. Hence $A x=x$.

Lemma 4.2. Let $G, G_{1}$ be connected Lie groups with $G_{1}$ a normal sub-

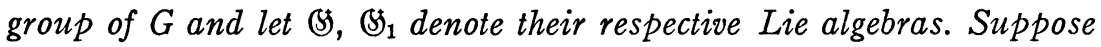

(a) $G_{1}$ is a Tychonoff group,

(b) $G / G_{1}$ is 1-dimensional,

(c) the adjoint representation of \&5 restricted to $\mathbb{B S}_{1}$ has matrices with only real eigenvalues. Then $G$ is a Tychonoff group.

Proof. We suppose that $G$ operates on a linear space $S$, that $V$ is a cone in $S$ with compact base, and that $L$ is a continuous linear functional on $S$, positive on $V$, and such that $\{x: L(x)=1\}$ intersects $V$ in a compact set. Since $G_{1}$ is a Tychonoff group, $G_{1}$ leaves fixed at least one ray of $V$. If $x$ is a vector on such a ray there is determined a positive character $\lambda$ on $G_{1}$ defined by $g_{1} x=\lambda\left(g_{1}\right) x$. Also, given a character $\lambda$, there will exist a cone (possibly empty) $V_{\lambda} \subset V$ of vectors $y \in V$ for which $g_{1} y=\lambda\left(g_{1}\right) y$. For $g \in G, g^{-1} G_{1} g \subset G_{1}$ and we may define the transform $\lambda^{g}$ of the character $\lambda$ by $\lambda^{g}\left(g_{1}\right)=\lambda\left(g^{-1} g_{1} g\right)$. If $x \in V_{\lambda}$,

$$
\lambda^{o}\left(g_{1}\right) g x=\lambda\left(g^{-1} g_{1} g\right) g x=g g^{-1} g_{1} g x=g_{1} g x,
$$

from which it follows that $g V_{\lambda} \subset V_{\lambda^{0}}$. Another consequence of (4.1) is that if $V_{\lambda}$ is nonempty, then $\lambda^{o}\left(g_{1}\right)$ is bounded as a function of $g$. For we have

$$
\begin{aligned}
\lambda^{o}\left(g_{1}\right) L(g x) & =L\left(g_{1} g x\right), \\
\lambda^{o}\left(g_{1}\right) & =L\left(g_{1} g x\right) / L(g x),
\end{aligned}
$$

and $L\left(g_{1} y\right) / L(y) \leqq \max L\left(g_{1} z\right)$, where $z$ ranges over the compact set of vectors in $V$ for which $L(z)=1$.

If $G_{1}^{\prime}$ denotes the commutator subgroup of $G_{1}$, then the group of positive characters on $G_{1}$ may be identified with the group of positive characters on $G_{1} / G_{1}^{\prime}$. Since the latter group is abelian, it is the image under the exponential map of its Lie algebra $\mathfrak{S}_{1} /\left[\mathfrak{G}_{1}, \mathfrak{G}_{1}\right]$. Thus the 
positive character group of $G_{1}$ may be identified with a subspace $W$ of the dual space $\left(\mathfrak{S}_{1} /\left[\mathfrak{G}_{1}, \mathfrak{S}_{1}\right]\right)^{*}$. Now it may be checked that the automorphism $\lambda \rightarrow \lambda^{\circ}$ of the positive character group of $G_{1}$ corresponds to the operation $X \rightarrow \operatorname{Ad} g(X)$ on $B_{1}$. Since ad $\$$ restricted to $\oiint_{1}$ has all real eigenvalues, its image under the exponential map, namely Ad $G$, has all its eigenvalues positive. This remains true when we pass to the quotient space $\mathfrak{S}_{1} /\left[\mathfrak{S}_{1}, \mathfrak{S}_{1}\right]$ of $\mathfrak{S}_{1}$, and to its dual, and to the subspace $W$ of the dual.

We see then that the set of positive characters on $G_{1}$ forms a linear space on which we have a representation of $G$ given by $\lambda \rightarrow \lambda^{o}$, and the eigenvalues of the transformations of this representation are all positive.

We know that for some $\lambda_{0}, V_{\lambda_{0}}$ is nonempty. This means by our previous observation that $\lambda_{0}^{0}\left(g_{1}\right)$ is bounded as a function of $g \in G$. But since the map $\lambda \rightarrow \lambda^{o}$ has positive eigenvalues, we see by Lemma 4.1 that $\lambda_{0}^{o}=\lambda_{0}$. If we also recall that $g V_{\lambda_{0}} \subset V_{\lambda_{0}^{g}}$, we find that $G$ takes $V_{\lambda_{0}}$ into itself. We shall now show that some ray of $V_{\lambda_{0}}$ is left fixed by all of $G$.

Let $V_{\lambda_{0}}^{\prime}$ denote the set of vectors $x \in V_{\lambda_{0}}$ with $L(x)=1$. If for each $g \in G$ we define $g^{*}$ by

$$
g^{*}(x)=g x / L(g x)
$$

then each $g^{*}$ takes $V_{\lambda_{0}}^{\prime}$ into itself. $g \rightarrow g^{*}$ is a homomorphism of $G$ into a group of continuous transformations of $V_{\lambda_{0}}^{\prime}$. However, $G_{1}^{*}$ leaves $V_{\lambda_{0}}^{\prime}$ pointwise fixed since each $g_{1} x$ is proportional to $x$ for $x \in V_{\lambda_{0}}^{\prime}$. Thus the action of $G^{*}$ on $V_{\lambda_{0}}^{\prime}$ is that of a one-parameter group. As a result $G^{*}$ has a fixed point and this means that $G$ has a fixed ray in $V_{\lambda_{0}}$. This completes the proof of the lemma.

CoRollary. A connected subgroup of the group of upper triangular real $r \times r$ matrices is a Tychonoff group.

PRoof. Such a group is solvable and we can find a chain $G=G_{0} \supset G_{1}$ $\supset G_{2} \ldots \supset G_{n}=e$ with $G_{i} / G_{i+1}$ 1-dimensional and $G_{i+1}$ normal in $G_{i}$. Let $Q$ denote the group of upper triangular matrices with positive entries on the diagonal. Since $G$ is connected, $G \subset Q$. If $\mathfrak{Q}$ is the Lie algebra of $Q$, then $\mathfrak{O}$ consists of real triangular matrices. For $q \in \mathfrak{Q}$, the eigenvalues of ad $q$ are differences of diagonal entries of $q$, and so they are real. This will be true for any subalgebra of $\mathfrak{Q}$ and so by Lemma 4.2 we may proceed inductively to prove that $G$ is a Tychonoff group.

Corollary. If $G$ is a semi-simple Lie group with an Iwasawa decomposition $G=K A N$, then $A N$ is a Tychonoff group. 
Proof. According to [11, Exposé 11, p. 14], $A N$ may be represented (faithfully) by upper triangular matrices.

THEOREM 4.1. If $G$ is a semi-simple Lie group, the subgroup $T$ defined at the end of $\$ 3$ is a Tychonoff group.

Proof. We recall that $T=K_{0} A N$ where $K_{0}$ is the centralizer of $A$ in $K$. Now since $N$ is the commutator subgroup of $A N$, every positive character $\lambda$ on $A N$ is identically 1 on $N$, and is determined by its values on $A$. If $k_{0} \in K_{0}$, then $\lambda^{k_{0}}(a)=\lambda\left(k_{0}^{-1} a k_{0}\right)=\lambda(a)$ for $a \in A$, so that $\lambda^{k_{0}}=\lambda$. Hence $k_{0} V_{\lambda} \subset V_{\lambda^{k_{0}}}=V_{\lambda}$, where $V_{\lambda}$ denotes the subset of $x$ in a cone $V$ (with compact base) with $g x=\lambda(g) x$ for $g \in A N$. Since $A N$ is a Tychonoff group, $V_{\lambda}$ will be nonempty for some $\lambda$. Let $x_{0} \in V_{\lambda}$ and form

$$
x=\int_{K_{0}} k_{0} x_{0} d k_{0} .
$$

Since $k_{0} V_{\lambda} \subset V_{\lambda}, x \in V_{\lambda}$, and so $A N$ leaves fixed the ray to which $x$ belongs. But clearly $K_{0}$ leaves $x$ fixed so that the ray to which $x$ belongs is left fixed by all of $T$.

By [6, Theorem 1.10] it follows that the subgroup $T$ is maximal with respect to the property of being a Tychonoff subgroup of $G$.

5. Spherical and semi-spherical functions. Before obtaining our generalization of the Choquet-Deny theorem, we shall discuss a special case connected with a class of functions which we shall call semispherical. Throughout this section, $G$ is a semi-simple group, $K$ a fixed maximal compact subgroup, $T$ the Tychonoff subgroup defined in $\S 3$ and $B(G)=G / T$. If $\gamma \in G$, let $A_{\gamma}$ denote the operator

$$
A_{\gamma} f(g)=\int_{\mathbf{K}} f(\gamma k g) d k=m_{K} * \gamma^{-1} * f(g) .
$$

Definition 5.1. A positive function $f$ on $G$ is semi-spherical if it is an eigenfunction of each $A_{\gamma}, \gamma \in G$.

The reason for our terminology is that a spherical function fulfills this condition, as we see from equation (3.2). But whereas a spherical function is bi-invariant under $K: \phi\left(k_{1} g k_{2}\right)=\phi(g)$, a semi-spherical function need only satisfy $f(k g)=f(g)$. (In fact, it is not difficult to show that a semi-spherical function which is bi-invariant under $K$ is actually spherical.)

If $f$ is semi-spherical, there is defined a function $\phi(\gamma)$ on $G$ by $A_{\gamma} f=\phi(\gamma) f$. We notice that $\phi(\gamma)$ is spherical: 


$$
\begin{aligned}
\int_{K} \int_{K} f\left(g_{1} k g_{2} k^{\prime} g_{3}\right) d k d k^{\prime} & =\int_{K} A_{\theta_{1}} f\left(g_{2} k^{\prime} g_{3}\right) d k^{\prime} \\
& =\phi\left(g_{1}\right) A_{g_{2}} f\left(g_{3}\right)=\phi\left(g_{1}\right) \phi\left(g_{2}\right) f\left(g_{3}\right) .
\end{aligned}
$$

But the first integral of (5.2) is also

$$
\int_{\boldsymbol{K}} A_{\theta_{1} k \sigma_{2}} f\left(g_{3}\right) d k=\left(\int_{\boldsymbol{K}} \phi\left(g_{1} k g_{2}\right) d k\right) f\left(g_{3}\right)
$$

and so

$$
\int_{K} \phi\left(g_{1} k g_{2}\right) d k=\phi\left(g_{1}\right) \phi\left(g_{2}\right),
$$

which is the defining equation of a spherical function.

Definition 5.2. We say a semi-spherical function $f$ belongs to the spherical function $\phi$ if

$$
\int_{\boldsymbol{K}} f(\gamma k g) d k=\phi(\gamma) f(g) ;
$$

the cone of semi-spherical functions belonging to $\phi$ will be denoted $V_{\phi}$.

$V_{\phi}$ is never empty since $\phi \in V_{\phi}$. Notice also that $V_{\phi}$ is closed under right translation, $R_{\gamma} V_{\phi} \subset V_{\phi}$, since the translates $R_{\gamma}$ commute with $A_{\gamma^{\prime}}$.

Lemma 5.1. If $\sigma \in M_{K}(X)$ where $X$ is a $G$-space such that $K$ is transitive on $X$, then the multiplier functions $\sigma(\cdot, x)$ are semi-spherical and belong to the spherical function

$$
\phi(g)=\int_{X} \sigma(g, x) d m(x) .
$$

Here $m$ denotes the unique $K$-invariant probability measure on $X$. ( $m$ is unique because $K$ is transitive on $X$.)

Proof.

$$
\begin{aligned}
\int \sigma(\gamma k g, x) d k & =\left(\int \sigma(\gamma k, g x) d k\right) \sigma(g, x) \\
& =\left(\int \sigma(\gamma, k g x) d k\right) \sigma(g, x) \\
& =\left(\int \sigma(\gamma, y) d m_{K} * g x(y)\right) \sigma(g, x) .
\end{aligned}
$$


Now the measure $m_{K} * g x$ is a $K$-invariant probability measure on $X$ and so $m_{K} * g x=m$. Thus

$$
A_{\gamma} \sigma(\cdot, x)=\phi(\gamma) \sigma(\cdot, x)
$$

which proves simultaneously that $\sigma$ is semi-spherical, that it belongs to $\phi$, and that the function $\phi$ defined by (5.4) is a spherical function.

Notice that this yields the easier half of the Harish-Chandra representation formula; namely, that the function defined by (3.4) is a spherical function. For we have shown that the integral in (3.4) is of the form (5.4) for the space $X=G / A N$.

We will show that the multiplier functions of Lemma 5.1 generate the set of all semi-spherical functions. More precisely, we will show that we may take $X=B(G)$ and each $V_{\phi}$ is spanned by the functions in $V_{\phi} \cap E_{K}(B(G))$.

To begin with we examine how the multiplier functions of $E_{K}(B(G))$ are expressed as measures. Suppose $\chi$ is a positive character on the group $T$, and form the measure $m_{T}^{\chi}$ on $T$ defined by

$$
d m_{T}^{x}(t)=\chi\left(t^{-1}\right) d t
$$

$d t$ denoting left invariant Haar measure on $T$. From (5.5) we obtain

$$
\operatorname{tm}_{T}^{x}=\chi(t) m_{T}^{x}
$$

for $t \in T$. Inasmuch as right-invariant Haar measure on $T$ is related to the left-invariant Haar measure by multiplication by a positive character, it follows that the measure $m_{T}^{\chi}$ is also related to rightinvariant Haar measure in a manner similar to (5.5). As a result we may also write

$$
m_{T}^{x} t=\chi^{\prime}(t) \stackrel{x}{x},
$$

where $\chi^{\prime}$ is some other positive character in $T$.

Now form the measure $m_{K} * m_{T}^{\chi}=\nu$. If $g=k t$, with $k \in K, t \in T$, then $m_{K} * g * m_{T}^{\chi}=\chi(t) m_{K} * m_{T}^{\chi}=\chi(t) \nu$. Since $K T=G$, every $g$ has such a decomposition. Viewing an arbitrary measure on $G$ as a linear combination of point measures we find that for any bounded measure $\omega$,

$$
m_{K} * \omega * m_{T}^{x}=c \nu
$$

for some constant $c$. From this it follows that $\nu$ is absolutely continuous, since $\omega$ could be chosen absolutely continuous. Let $f$ be the density of $\nu: d \nu(g)=f(g) d g$. Then

$$
f(k g)=f(g), \quad f(g t)=\chi^{\prime}\left(t^{-1}\right) f(g)
$$


by (5.7). We may therefore define a multiplier by

$$
\sigma(g, \gamma T)=f(g \gamma) / f(\gamma)
$$

since if $\gamma$ is replaced by $\gamma t$, both numerator and denominator are multiplied by $\chi^{\prime}\left(t^{-1}\right)$. It is, moreover, clear that $\sigma$ is a multiplier and is in $M_{K}(B(G))$. Thus the measure $m_{K} * m_{T}^{\chi}$, as a function, is proportional to the multiplier function $\sigma(g, T)$.

We shall need the following lemma whose proof we leave to the Appendix.

Lemma 5.2. If $\pi$ is a measure on $G$ satisfying $t \pi=\chi(t) \pi$ for $t \in T$, and $\chi$ a positive character on $T$, then $\pi=m_{T}^{\chi} * \mu$ for some positive measure $\mu$ on $G$.

We now turn to the proof of the fact that the $K$-multiplier functions for $B(G)$ span the cones $V_{\phi}$. Suppose $h \in V_{\phi}$. We then have

$$
m_{K} * \gamma^{-1} * h=\phi(\gamma) h .
$$

Form the cone $V^{\prime}$ of all positive measures $\pi$ with the property that $m_{K} * \gamma^{-1} * \pi$ is proportional to $h$ for all $\gamma \in G$. Then $h \in V^{\prime}$ and also $V^{\prime}$ is closed under translation from the left $\pi \rightarrow g \pi$. We claim that $V^{\prime}$ is a cone with compact base in the weak topology on measures. If $\psi$ is some positive function with compact support on $G$ with

$$
\int \psi(g) h(g) d g>0
$$

define a linear functional $L$ by

$$
L(\pi)=m_{K} * \pi(\psi) / \int \psi(g) h(g) d g .
$$

$L$ is continuous and the intersection $V_{1}^{\prime}$ of $V^{\prime}$ with the set of $\pi$ such that $L(\pi)=1$ consists of those $\pi$ in $V^{\prime}$ for which $m_{K} * \pi=h$. We claim this set is compact. Since it is closed, it suffices to show that for each compact set $J \subset G, \pi(J)$ is bounded as $\pi$ ranges over $V_{1}^{\prime}$. But

$$
m_{K} * \pi(K J)=\int_{K} \int_{G} C_{K J}(k g) d k d \pi(g),
$$

with $C_{K J}$ the indicator function of $K J$. But $C_{K J}(k g) \geqq C_{K}(k) C_{J}(g)$ so

$$
\int_{K J} h(g) d g \geqq m_{K}(K) \pi(J)=\pi(J),
$$

which gives the desired result. So $V^{\prime}$ is a cone with compact base. 
Since $G$ and therefore $T$ operates on $V^{\prime}$ on the left, and since $T$ is a Tychonoff group (Theorem 4.1), there is a measure $\pi \in V^{\prime}$ with $t \pi=\chi(t) \pi$ for all $t \in T$ and some positive character $\chi$. By Lemma 5.2, $\pi=m_{T}^{\mathrm{x}} * \mu$ for some measure $\mu$ on $G$. Thus for some $\chi$, the measure $m_{T}^{\chi} * \mu$ is in $V^{\prime}$. This means that $m_{K} * \gamma^{-1} * m_{T}^{\chi} * \mu$ is proportional to $h$ for every $\gamma$ and, in particular, $m_{K} * m_{T}^{\chi} * \mu$ is proportional to $h$. But $m_{K} * m_{T}^{x} \leftrightarrow \sigma\left(\cdot, x_{0}\right)$ for $\sigma \in M_{K}(B(G))$ and for $x_{0}$ the point of $B(G)$ corresponding to the coset T. $m_{K} * m_{T}^{\chi} * \mu$ is a linear combination of right translates of $m_{K} * m_{T}^{\chi}$ and so it follows that

$$
\begin{aligned}
h(g) & =\int_{G} \sigma\left(g \gamma, x_{0}\right) d \mu(\gamma)=\int_{G} \sigma\left(g, \gamma x_{0}\right) \sigma\left(\gamma, x_{0}\right) d \mu(\gamma) \\
& =\int_{B(G)} \sigma(g, x) d \mu^{*}(x)
\end{aligned}
$$

for some positive measure $\mu^{*}$ on $B(G)$. Now by Lemma $5.1, \sigma(\cdot, x)$ belongs to some spherical function $\phi^{\prime}$ defined by

$$
\phi^{\prime}(g)=\int_{B(G)} \sigma(g, x) d m(x),
$$

with $m$, as usual, denoting the unique $K$-invariant probability measure on $B(G)$. But then the function $h(g)$ satisfying (5.11) must belong to the same $\phi^{\prime}$. Our assumption, however, was that $h \in V_{\phi}$. We must, therefore, have $\phi^{\prime}=\phi$. We have thereby proven

THEOREM 5.1. Let $\phi$ be a positive spherical function and $V_{\phi}$ the cone of semi-spherical functions belonging to $\phi$. For each $h \in V_{\phi}$ there is a multiplier $\sigma \in M_{K}(B(G))$ with $\sigma(\cdot, x) \in V_{\phi}$ for each $x \in B(G)$ and such that

$$
h(g)=\int_{B(G)} \sigma(g, x) d \mu^{*}(x)
$$

for some positive measure $\mu^{*}$ on $B(G)$. The multiplier $\sigma$ which occurs in (5.12) must satisfy

$$
\phi(g)=\int_{B(G)} \sigma(g, x) d m(x),
$$

where $m$ is the unique $K$-invariant probability measure on $B(G)$.

It should be noticed that we have incidentally proven that every positive spherical function $\phi$ admits a representation (5.13), which is essentially the Harish-Chandra formula. 
If the $K$-multiplier functions $\sigma(\cdot, x)$ belong to $V_{\phi}$ we shall simply say that $\sigma$ belongs to $\phi$. Thus every spherical function has a $K$-multiplier belonging to it. In fact there is always a finite set of $K$-multipliers belonging to each spherical function, the cardinality of this set being, in general, equal to the order of the Weyl group of $G$. (See $[8$, Chapter X].) It will develop that even though a number of distinct $K$-multipliers (over $B(G)$ ) belong to $\phi$, only one of them is needed to span $V_{\phi}$.

6. The Choquet-Deny representation. The considerations of this section are based on [3]. We are interested in determining when the points of a convex cone can be expressed as resultants of measures placed on the extremal points of the cone. We call a point $x$ of a cone $V$ an extremal point if it lies on an extremal ray, namely, if $y \in V$ and $x-y \in V$ imply $y=\lambda x$ for $\lambda \leqq 1$. We shall be concerned with cones of positive (unbounded) measures on a separable locally compact space $X$. We regard the space of such measures as the dual to the space $C^{0}$ of continuous functions of compact support on $X$ and we endow it with the corresponding weak topology. Suppose $V$ is a closed cone of positive measures on $X$ and suppose $\mu$ is a measure on $V$. We form the resultant $\bar{\mu}$ by setting

$$
\bar{\mu}(\psi)=\int \pi(\psi) d \mu(\pi),
$$

where $\psi$ is a function of compact support, and the integral on the right is assumed to exist. When it does, the resultant $\bar{\mu}$ is again a measure in $V$. First of all, it is a positive measure because (6.1) defines a positive linear functional on $C^{0}$. Also $\bar{\mu} \in V$ for if $\bar{\mu} \notin V$ there would exist, by the Hahn-Banach theorem, a function $\psi \in C^{0}$ with $\bar{\mu}(\psi)<0$ and $\pi(\psi) \geqq 0$ for all $\pi \in V$. This is impossible by (6.1).

In general a cone $V$ is a lattice if whenever $x_{1}, x_{2} \in V$, there exists an infimum $y=\inf \left(x_{1}, x_{2}\right)$ with respect to the ordering of $V$. That is, there exists $y \in V$ with $x_{1}-y, x_{2}-y \in V$, and if, for some other $y^{\prime} \in V, x_{1}-y^{\prime}, x_{2}-y^{\prime} \in V$, then $y-y^{\prime} \in V$.

The result we shall need is the following:

THEOREM 6.1. Let $V$ be a weakly closed cone of positive measures on a separable, locally compact space $X$ and let $\mathcal{E}$ denote the extremal rays of $V$. Suppose there is a positive function of compact support $\psi$ with $\pi(\psi)>0$ for all $\pi \in V, \pi \neq 0$, and let $V_{1}=\{\pi: \pi(\psi)=1\}$. Then for each $\pi \in V$ there exists a measure $\mu$ on a Borel subset of $\varepsilon \cap V_{1}$ such that $\pi$ is the resultant $\bar{\mu}$. If, moreover, $V$ is a lattice, then the measure $\mu$ is unique. 
Clearly if we wish to have a unique representation in a certain set, then this set can intersect each ray in at most one point. That is the reason for restricting the representation to $V_{1}$.

The proof of Theorem 6.1 is based on the corresponding result for compact, convex sets. If $W$ is a compact, convex set it is possible to define the resultant $\bar{\mu}$ of a probability measure $\mu$ on $W$, and $\bar{\mu}$ will be a point of $W$. We then have [2], [9]

TheOREM A. If $W$ is metrizable then the set of extremals of $W$ is a Borel set and every point of $W$ is the resultant of some measure on the set of extremals of $W$.

A convex set $W$ may be taken to be the base of a convex cone. For example, if $W$ is in the linear space $S$ we may form the cone $\tilde{W}$ in $R \times S$ by setting $\tilde{W}=\{(t, t w): t>0, w \in W\}$. We say that $W$ is a simplex if $\tilde{W}$ is a lattice.

ThEOREM B [2], [9]. If $W$ is metrizable and is a simplex, then each point of $W$ is the resultant of a unique probability measure on the set of extremals of $W$.

We apply these theorems to the cone $V$ of Theorem 6.1 by showing that each point of $V$ lies in some compact convex subset $W \subset V$. Let $\pi \in V$. By the separability of $X$ there will be some continuous function $h$ everywhere positive in $X$ and such that $\pi(h)<\infty$. Suppose $\pi(h)=1$. Let $W$ be the set of $\pi^{\prime} \in V$ with $\pi^{\prime}(h) \leqq 1$. This set is closed in $V$ and moreover, since $h(x)>0$ everywhere, so that on every compact set $h(x)$ is bounded from below, the measures $\pi^{\prime}$ in $W$ are bounded in every compact subset of $X$. Thus $W$ is a compact convex metrizable set. Let us show that each extremal of $W$ with the exception of the point 0 is an extremal of $V$. If $\pi^{\prime}$ is an extremal of $W$ and $\pi^{\prime}=\pi_{1}^{\prime}+\pi_{2}^{\prime}$ with $\pi_{i}^{\prime} \in V$, then $\pi_{i}^{\prime}(h)>0$ unless $\pi_{i}^{\prime}=0$. So

$$
\pi^{\prime}=\pi_{1}^{\prime}(h) \frac{\pi_{1}^{\prime}}{\pi_{1}^{\prime}(h)}+\pi_{2}^{\prime}(h) \frac{\pi_{2}^{\prime}}{\pi_{2}^{\prime}(h)},
$$

which expresses $\pi^{\prime}$ as a convex combination of measures in $W$ since $\pi_{1}^{\prime}(h)+\pi_{2}^{\prime}(h)=1$. It follows that $\pi_{1}^{\prime}$ and $\pi_{2}^{\prime}$ are proportional to $\pi^{\prime}$. Now by Theorem A, $\pi$ is the resultant of a measure on the extremal points of $W$ :

$$
\pi=\int \pi^{\prime} d \mu\left(\pi^{\prime}\right)=\int \frac{\pi^{\prime}}{\pi^{\prime}(\psi)} \pi^{\prime}(\psi) d \mu\left(\pi^{\prime}\right)
$$

and this expresses $\pi$ as the resultant of a measure on $\varepsilon \cap V_{1}$. 
We observe that conversely, if $\pi$ is given as the resultant of a measure on $\varepsilon \cap V_{1}$, then this measure corresponds to one on the extremals of $W$. Namely, if

$$
\pi=\int \pi^{\prime} d \mu\left(\pi^{\prime}\right)
$$

then $\int \pi^{\prime}(h) d \mu\left(\pi^{\prime}\right)=1$ and $\pi^{\prime}(h)<\infty$ for almost all $\pi^{\prime}$ with respect to $\mu$. We can then write

$$
\pi=\int \frac{\pi^{\prime}}{\pi^{\prime}(h)} \pi^{\prime}(h) d \mu\left(\pi^{\prime}\right)
$$

and $\pi^{\prime}(h) d \mu\left(\pi^{\prime}\right)$ is a probability measure, and the measures $\pi^{\prime} / \pi^{\prime}(h)$ are extremals of $W$ if the $\pi^{\prime}$ are extremals of $V$. As a result of this observation it follows that to prove the uniqueness portion of Theorem 6.1 it suffices, in view of Theorem B, to show that if $V$ is a lattice then $W$ is a simplex. Equivalently we must show that $\tilde{W}$ is a lattice. This follows readily. If $x_{1}=\left(t, t \pi_{1}\right), x_{2}=\left(s, s \pi_{2}\right)$ are two points of $\tilde{W}$ where $\pi_{1}, \pi_{2} \in W$, then if $t<s$, we set

$$
y=\inf _{\tilde{W}}\left(x_{1}, x_{2}\right)=\left(t, t \inf _{V}\left(\pi_{1}, \frac{s}{t} \pi_{2}\right)\right) .
$$

Notice that $y \in \tilde{W}$ inasmuch $\operatorname{as}_{V}\left(\pi_{1}, s \pi_{2} / t\right)$ is in $W$ (being bounded by $\left.\pi_{1} \in W\right)$. If we rewrite $y$ as

$$
\left(t, \inf _{V}\left(t \pi_{1}, s \pi_{2}\right)\right)
$$

it becomes clear that $x_{1}-y, x_{2}-y \in \tilde{W}$ and if $x_{1}-y^{\prime}, x_{2}-y^{\prime} \in \tilde{W}$ then $y-y^{\prime} \in \tilde{W}$.

The uniqueness of the representation in Theorem 6.1 for the case that $V$ is a lattice may also be formulated as follows:

CoRollary. If $V$ is a cone of measures as in Theorem 6.1 and $V$ is a lattice, then if $\nu$ is a signed measure on $\mathcal{E} \cap V_{1}$ such that $\int \pi^{\prime} d \nu\left(\pi^{\prime}\right) \in V$, then $\nu \geqq 0$.

Proof. Write $\nu=\nu_{1}-\nu_{2}$ with $\nu_{i} \geqq 0$ and suppose that $\int \pi^{\prime} d \nu\left(\pi^{\prime}\right)$ $=\int \pi^{\prime} d \nu_{3}\left(\pi^{\prime}\right)$ with $\nu_{3} \geqq 0$. Then $\bar{\nu}_{1}=\bar{\nu}_{2}+\bar{\nu}_{3}$ and, by uniqueness, $\nu_{1}$ $=\nu_{2}+\nu_{3}$. Then $\nu=\nu_{3} \geqq 0$.

Theorem 6.1 is used to prove the following result which we refer to as the Choquet-Deny representation.

THEOREM 6.2. Let $G$ be a separable locally compact group and suppose that $\left\{\mu_{\alpha}, \alpha \in \Gamma\right\}$ is a commuting family of positive measures with com- 
pact supports on $G\left(\mu_{\alpha} * \mu_{\beta}=\mu_{\beta} * \mu_{\alpha}, \alpha, \beta \in \Gamma\right)$. Let $V$ denote the set of positive measures $\pi$ on $G$ satisfying $\mu_{\alpha} * \pi=\pi$ for all $\alpha \in \Gamma$. Assume moreover that there exists a continuous function of compact support $\psi \geqq 0$ on $G$ with $\pi(\psi)>0$ for all $\pi \in V, \pi \neq 0$. If $\mathcal{E}$ denotes the set of extremals of $V$ and $V_{1}=\{\pi: \pi(\psi)=1\}$, then, for each $\pi \in V$ there exists a unique Borel measure $\nu_{\pi}$ on $\varepsilon \cap V_{1}$ with $\pi=\bar{\nu}_{\pi}$.

Proof. Since the measures $\mu_{\alpha}$ have compact support it follows that $V$ is closed. Thus the existence of a measure $\nu_{\pi}$ is guaranteed by the first part of Theorem 6.1. To prove uniqueness we must show that $V$ is a lattice. Suppose then that $\pi_{1}, \pi_{2} \in V$. Denote by $\pi^{\prime}$ the infimum of $\pi_{1}$ and $\pi_{2}$ in the lattice of all positive measures. $\pi^{\prime}$ may be defined by

$$
\frac{d \pi^{\prime}}{d\left(\pi_{1}+\pi_{2}\right)}(g)=\min \left(\frac{d \pi_{1}}{d\left(\pi_{1}+\pi_{2}\right)}(g), \frac{d \pi_{2}}{d\left(\pi_{1}+\pi_{2}\right)}(g)\right) .
$$

Let $S$ denote the convolution semigroup of measures on $G$ generated by the $\mu_{\alpha}, \alpha \in \Gamma$. We shall consider the family $S * \pi^{\prime}$ of measures $\mu * \pi^{\prime}, \mu \in S$. We have

$$
\mu_{\alpha} * \pi^{\prime} \leqq \mu_{\alpha} * \pi_{i}=\pi_{i}, \quad i=1,2,
$$

so that $\mu_{\alpha} * \pi^{\prime} \leqq \pi^{\prime}$. Hence $\mu * \pi^{\prime} \leqq \pi^{\prime}$ for any $\mu \in S$. By the commutativity of $S$ we also have

$$
\mu^{\prime} *\left(\mu * \pi^{\prime}\right) \leqq \mu * \pi^{\prime}
$$

for $\mu, \mu^{\prime} \in S$.

Choose a dense subset $\left\{\mu_{n} * \pi^{\prime}\right\}$ in $S * \pi^{\prime}$. This may be done because $G$ is separable. Let

$$
\pi=\lim _{n \rightarrow \infty} \mu_{1} * \cdots * \mu_{n} * \pi^{\prime}
$$

This limit exists because the sequence of measures is decreasing by (6.2). Clearly $\pi \leqq \mu * \pi^{\prime}$ for any $\mu \in S$. Also $\mu_{\alpha} * \pi \leqq \pi$. But $\mu_{\alpha} * \pi$ is a limit of measures in $S * \pi^{\prime}$ and so $\pi \leqq \mu_{\alpha} * \pi$. Hence $\pi=\mu_{\alpha} * \pi$ and $\pi \in V$. Since $\pi \leqq \pi^{\prime}$ we have $\pi_{1}-\pi \in V$ and $\pi_{2}-\pi \in V$. Now suppose $\pi_{1}-\tilde{\pi}, \pi_{2}-\tilde{\pi} \in V$ for some $\tilde{\pi} \in V$. Then $\tilde{\pi} \leqq \pi^{\prime}$ and $\mu * \tilde{\pi} \leqq \mu * \pi^{\prime}$ for all $\mu \in S$. But if $\tilde{\pi} \in V$ then $\mu * \tilde{\pi}=\tilde{\pi}$, so $\tilde{\pi} \leqq \mu * \pi^{\prime}$ for $\mu \in S$, and so $\tilde{\pi} \leqq \pi$ or $\pi-\tilde{\pi} \in V$. Therefore $\pi=\inf \left(\pi_{1}, \pi_{2}\right)$ with respect to $V$. This proves the theorem.

7. Application to semi-spherical functions. We apply the foregoing to obtain more information regarding the representation of semispherical functions in terms of multiplier functions. In particular we shall see that for each spherical function $\phi(\phi>0)$ there is a single 
$K$-multiplier $\sigma_{\phi} \in M_{K}(B(G))$ such that $V_{\phi}$ is spanned by $\sigma_{\phi}(\cdot, x) . G$ is again a semi-simple Lie group.

Leмma 7.1 [7], [8]. The measures $m_{K} * g * m_{K}$ for $g \in G$ commute with one another.

Proof. We use the fact that there is a map $g \rightarrow g^{\prime}$ of $G$ into itself with the following properties: (a) $\left(g^{\prime}\right)^{\prime}=g$, (b) $\left(g_{1} g_{2}\right)^{\prime}=g_{2}^{\prime} g_{1}^{\prime}$, (c) $k^{\prime}=k^{-1}$ for $k \in K$, (d) if $P=\left\{g: g^{\prime}=g\right\}$ then $K P=P K=G$. (In case $G=\operatorname{SL}(n, R), g^{\prime}$ denotes transposition.)

We extend the operation $g \rightarrow g^{\prime}$ to measures $\mu \rightarrow \mu^{\prime}$. We then have $m_{K}^{\prime}=m_{K}$ and $\left(m_{K} * p * m_{K}\right)^{\prime}=m_{K} * p * m_{K}$ for $p \in P$. Now every $g \in G$ may be expressed as $g=k p$ for $k \in K, p \in P$ and $m_{K} * g * m_{K}$ $=m_{K} * p * m_{K}$. It follows that $\left(m_{K} * g * m_{K}\right)^{\prime}=m_{K} * g * m_{K}$. From this it follows that generally $\left(m_{K} * \mu * m_{K}\right)^{\prime}=m_{K} * \mu * m_{K}$. So $m_{K} * g_{1} * m_{K} * m_{K} * g_{2} * m_{K}$ is invariant under ( $)^{\prime}$, but, on the other hand, using (b) it must equal $\left(m_{K} * g_{2} * m_{K}\right)^{\prime} *\left(m_{K} * g_{1} * m_{K}\right)^{\prime}$ $=m_{K} * g_{2} * m_{K} * m_{K} * g_{1} * m_{K}$. This proves the lemma.

Now the functions in $V_{\phi}$ are the solutions $\geqq 0$ of

$$
m_{K} * \gamma^{-1} * f=\phi(\gamma) f \text {. }
$$

The measure solutions of (7.1) are actually functions because $f$ is proportional to $m_{K} * \mu * f$ for any $\mu$, in particular, an absolutely continuous one. Since $m_{K} * f=f$ we may rewrite (7.1) as

$$
\phi(\gamma)^{-1} m_{K} * \gamma^{-1} * m_{K} * f=f \text {. }
$$

Since the measures $m_{K} * \gamma^{-1} * m_{K}$ are mutually commutative and of compact support, the cone $V_{\phi}$ of solutions to (7.2) is one to which Theorem 6.2 applies. Moreover, a function $f \in V_{\phi}$ must be everywhere positive unless $f \equiv 0$, since by

$$
\int_{\boldsymbol{K}} f(\gamma k g) d k=\phi(\gamma) f(g) ;
$$

if $f(g)=0$, since $f$ is continuous, $f$ must vanish everywhere. Hence for any function $\psi \geqq 0$ of nonempty compact support, $\int f(g) \psi(g) d g>0$. Choose $\psi$ so that $\psi(g k)=\psi(g)$ for $k \in K$ and $\int \psi(g) \phi(g) d g=1$. Then

$$
\begin{aligned}
\int_{G} f(g) \psi(g) d g & =\int_{K} \int_{G} f(g k) \psi(g) d g d k \\
& =\int_{G} \phi(g) \psi(g) d g f(e)=f(e) .
\end{aligned}
$$

The set $V_{1}$ of Theorem 6.2 is therefore the set of functions with unit 
value at the identity. We refer to $\mathcal{E} \cap V_{1}$ as the normalized extremals. Theorem 6.2 then asserts that each semi-spherical function in $V_{\phi}$ has a unique representation in terms of normalized extremals of $V_{\phi}$. Now by Theorem 5.1, every function in $V_{\phi}$ is an integral over multiplier functions $\sigma(\cdot, x)$ in $V_{\phi}$ (equation (5.12)). It follows that an extremal of $V_{\phi}$ must already be proportional to a multiplier function. Since the multiplier functions satisfy $\sigma(e, x)=1$, we see that the normalized extremals of $V_{\phi}$ are multiplier functions.

We next show that only one $K$-multiplier $\sigma_{\phi} \in M_{K}(B(G))$ can be such that the functions $\sigma_{\phi}(\cdot, x)$ are extremals of $V_{\phi}$. Suppose that $\sigma_{1}(\cdot, x)$ and $\sigma_{2}(\cdot, x)$ are all extremals in $V_{\phi}$. (Note that if $\sigma_{1}\left(\cdot, x_{1}\right)$ is an extremal so is $R_{\gamma} \sigma_{1}\left(\cdot, x_{1}\right)=\sigma_{1}\left(\cdot, \gamma x_{1}\right) \sigma_{1}\left(\gamma, x_{1}\right)$ so that all $\sigma_{1}(\cdot, x)$ are extremals if one of them is.) Now if $\sigma_{1}(\cdot, x) \in V_{\phi}$ then $\int_{B(G)} \sigma_{1}(g, x) d m(x)$ $=\phi(g)$. The same holds for $\sigma_{2}$. But $\phi \in V_{\phi}$ has a unique representation in terms of its extremals (Theorem 6.2) and so the set of functions $\sigma_{1}(\cdot, x)$ must be identical with the set $\sigma_{2}(\cdot, x)$. Now both these sets of functions $B_{1}$ and $B_{2}$ form $G$-spaces with the operations $h \rightarrow R_{g} h / h(g)$. Moreover it is easy to see that $x \rightarrow \sigma_{i}(\cdot, x)$ then gives an equivariant map of $B(G)$ onto $B_{i}, i=1,2$. But the spaces $B_{i}$ are the same, so if $\sigma_{1} \neq \sigma_{2}$, we obtain two distinct equivariant maps of $B(G)$ onto $B_{1}=B_{2}$.

LemMa 7.2. If $j_{1}: B(G) \rightarrow B, j_{2}: B(G) \rightarrow B$ are two equivariant maps of $B(G)$ onto the same space $B$, then $j_{1}=j_{2}$.

PROOF. It suffices to show that for some point $x_{0} \in B(G), j_{1}\left(x_{0}\right)$ $=j_{2}\left(x_{0}\right)$. For any $x \in B(G)$ form the measure $\pi=\frac{1}{2}\left\{\delta_{j_{1}(x)}+\delta_{j_{2}(x)}\right\}$ on $B$. Since $B$ is a boundary (end of $\S 3$ ), there exists a sequence $\left\{g_{n}\right\} \subset G$ with $g_{n} \pi$ converging to a point measure. But extracting a subsequence we will have $g_{n_{k}} x \rightarrow x_{0}$ for some $x_{0} \in B(G)$ and so $g_{n_{k}} \pi \rightarrow \frac{1}{2}\left\{\delta_{j_{1}\left(x_{0}\right)}+\delta_{j_{2}\left(x_{0}\right)}\right\}$. If this is a point measure we must have $j_{1}\left(x_{0}\right)=j_{2}\left(x_{0}\right)$. Hence $j_{1}=j_{2}$.

It follows by this lemma that the maps $x \rightarrow \sigma_{i}(\cdot, x)$ must be the same for $i=1,2$, and so $\sigma_{1}=\sigma_{2}$. We have thereby proved

THEOREM 7.1. To each positive spherical function $\phi$ on $G$ there is a multiplier $\sigma_{\phi} \in M_{K}(B(G)$ ) such that every positive solution to

$$
\int_{\boldsymbol{K}} f(\gamma k g) d k=\phi(\gamma) f(g)
$$

is given by

$$
f(g)=\int_{B(G)} \sigma_{\phi}(g, x) d \nu(x),
$$

where $\nu$ is a positive measure on $B(G)$. 
For, the function $f \in V_{\phi}$ has a representation in terms of normalized extremals of $V_{\phi}$. We have seen that these extremals must be multiplier functions and, moreover, only one multiplier belonging to $\phi$ can give rise to extremals. We denote that multiplier by $\sigma_{\phi}$.

There is still one improvement that may be made on Theorem 7.1. We know that a measure on the set of extremals of $V_{\phi}$ is uniquely determined by its resultant, but we cannot as yet identify $B(G)$ with the set of normalized extremals. Namely the map $x \rightarrow \sigma_{\phi}(\cdot, x)$ may not be $1-1$. We shall see later that this map is $1-1$, so that the measure $\nu$ in (7.4) is uniquely determined by the function $f$.

According to Lemma 5.1, every multiplier function in $E_{K}(B(G))$ is semi-spherical and belongs to some $V_{\phi}$. We may then apply Theorem 7.1 to obtain an integral representation of any multiplier function with respect to $B(G)$ in terms of some $\sigma_{\phi}$. Even in the simplest cases this leads to nontrivial relationships.

Let us illustrate this for the case of the group $G$ of analytic automorphisms of the unit disc. In the Iwasawa decomposition of this group $G$, the vector group is 1-dimensional. It follows that $M_{K}(B(G))$ is 1-dimensional $(\sigma(g, \gamma T)$ is determined by the positive character $\sigma(t, T)$ which is determined by $\sigma(a, T))$. For this group, $B(G)$ is the boundary of the unit disc, $B(G)=\left\{e^{i \theta}\right\}$. We have seen in $\S 3$ that $\left|g^{\prime}\left(e^{i \theta}\right)\right|$ is a $K$-multiplier with respect to $B(G)$ and so it follows that all the $K$-multipliers in $M_{K}(B(G))$ are given by

$$
\sigma_{s}\left(g, e^{i \theta}\right)=\left|g^{\prime}\left(e^{i \theta}\right)\right| s, \quad-\infty<s<\infty .
$$

If we denote by $m$ normalized Lebesgue measure on the unit circle, then (7.5) can also be written as

$$
\sigma_{s}\left(g, e^{i \theta}\right)=\left[\frac{d g^{-1} m}{d m}\left(e^{i \theta}\right)\right]^{s} .
$$

We recall from $\$ 3$ that this expression always represents a multiplier. The spherical functions on $G$ and therefore given by

$$
\phi_{s}(g)=\int \sigma_{s}\left(g, e^{i \theta}\right) d m\left(e^{i \theta}\right) .
$$

Now, in general, if $G$ is a semi-simple group and $B(G)$ is its associated $G$-space we can form a 1-parameter family of spherical functions by taking

$$
\phi_{s}(g)=\int_{B(G)}\left[\frac{d g^{-1} m}{d m}(x)\right]^{s} d m(x), \quad-\infty<s<\infty .
$$

We then have 


$$
\begin{aligned}
\phi_{1-s}(g) & =\int_{B(G)}\left[\frac{d g^{-1} m}{d m}(x)\right]^{-8} \frac{d g^{-1} m}{d m}(x) d m(x) \\
& =\int_{B(G)}\left[\frac{d g^{-1} m}{d m}(x)\right]^{-s} d g^{-1} m(x) \\
& =\int_{B(G)}\left[\frac{d g^{-1} m}{d m}\left(g^{-1} x\right)\right]^{-8} d m(x) \\
& =\int_{B(G)}\left[\frac{d m}{d g m}(x)\right]^{-s} d m(x) \\
& =\int_{B(G)}\left[\frac{d g m}{d m}(x)\right]^{s} d m(x)=\phi_{s}\left(g^{-1}\right) .
\end{aligned}
$$

In our case it can also be verified that $\phi_{s}(g)=\phi_{s}\left(g^{-1}\right)$. (Every $g \in G$ can be written $g=k_{1} h k_{2}$ where $k_{1}$ and $k_{2}$ are rotations and

$$
h(z)=\frac{z-\tau}{1-\tau z}, \quad-1<\tau<1 .
$$

But $h=h^{-1}$, so $\phi_{s}(g)=\phi_{s}(h)=\phi_{s}\left(h^{-1}\right)=\phi_{s}\left(g^{-1}\right)$.) For this group it follows that $\sigma_{s}$ and $\sigma_{1-s}$ belong to the same spherical function. As a result, for each $s$ we must be able to express $\sigma_{s}\left(\cdot, e^{i \theta}\right)$ in terms of $\sigma_{1-s}\left(\cdot, e^{i \theta}\right)$, or vice versa. Now it can be seen that $\sigma_{1 / 2+t}$ grows more rapidly than $\sigma_{1 / 2-t}$ and, as a result, the latter must be expressed in terms of the former. Granting that such an expression exists, it is not difficult to determine what it must be. If we write $\sigma_{1-s}(g, 1)$ $=\int \sigma_{s}\left(g, e^{i \theta}\right) d \nu(\theta)$, and use the multiplier property for $\sigma_{1-s}$ and $\sigma_{s}$ and the uniqueness of the decomposition in terms of $\sigma_{s}(\cdot, x)$-the extremal of $V_{\phi_{s}}$-we conclude that

$$
\frac{d t \nu}{d \nu}\left(e^{i \theta}\right)=\sigma_{1-s}(t, 1) \sigma_{s}\left(t^{-1}, e^{i \theta}\right)
$$

for $t \in G$ and $t 1=1$. This determines the measure $\nu$. If we write

$$
\sigma_{s}\left(g, e^{i \theta}\right)=\left(\frac{1-r^{2}}{1-2 r \cos (\theta-\phi)+r^{2}}\right)^{s}=P(r, \theta-\phi)^{s},
$$

the relationship in question is

$$
\begin{aligned}
P(r, \theta)^{1-s} & =\gamma_{s} \int_{0_{e}}^{2 \pi} P(r, \theta-\phi)^{s}(1-\cos \phi)^{s-1} d \phi \\
\gamma_{s} & =\left[\int_{0}^{2 \pi}(1-\cos \phi)^{s-1} d \phi\right]^{-1} .
\end{aligned}
$$


(7.8) is proved by these means to be true for $s>1 / 2$. Since both sides of (7.8) are analytic for $R s>1 / 2$, the same formula holds for these complex values as well. Apparently a limiting form of (7.8) is still valid for $R s=1 / 2$. In this case the kernel $(1-\cos \phi)^{1 / 2+i t}$ is singular, but with the proper interpretation it defines a unitary operator on $L^{2}(0,2 \pi)$. Equation $(7.8)$ then shows that this operator is the intertwining operator relating the equivalent unitary representations obtained from the complex multipliers $\sigma_{1 / 2+i t}$ and $\sigma_{1 / 2-i t}$ (see $\$ 2$ ).

8. The general convolution equation. We now come to our main result which deals with solutions to the general convolution equation

$$
\int f\left(g^{\prime} g\right) d \mu\left(g^{\prime}\right)=\lambda f(g), \quad \lambda>0 .
$$

Unfortunately, we shall not be able to handle the case of an arbitrary positive measure $\mu$. We shall have to assume, to begin with, that $\mu$ is absolutely continuous and we shall also make a special assumption regarding the support of $\mu$. Specifically, we shall take $d \mu(g)=\psi(g) d g$ where $\psi(g)$ is a bounded measurable function of compact support $\Delta$, and assume that $\Delta$ has the property that some power $\Delta^{n}$ contains a neighborhood of the identity. These hypotheses regarding $\psi$ will be assumed in force throughout our discussion. Our equation now takes the form

$$
\int f\left(g^{\prime} g\right) \psi\left(g^{\prime}\right) d g^{\prime}=\lambda f(g)
$$

and we denote by $V_{\lambda}(\psi)$ the cone of non-negative solutions to (8.2). We shall show that $V_{\lambda}(\psi)$ is spanned by its extremals and that the extremals are multiplier functions. Note that unlike equation (5.3), the solutions to (8.2) need not satisfy $f(k g)=f(g)$. As a result, the multiplier functions that we deal with now are no longer $K$-multiplier functions. As usual, $V_{\lambda}(\psi)$ is translation invariant, and so, for a multiplier $s \in M(B(G))$, if $s\left(\cdot, x_{1}\right) \in V_{\lambda}(\psi)$ for some $x_{1} \in B(G)$, then $s(\cdot, x) \in V_{\lambda}(\psi)$ for all $x \in B(G)$.

Lemma 8.1. In each equivalence (cohomology) class of $H(B(G)$ ) there is at most one multiplier $s$ with $s(\cdot, x) \in V_{\lambda}(\psi)$.

Proof. Let us denote by $\psi^{(n)}$ the $n$-fold convolution of $\psi$ with itself. Notice that under our hypotheses, $\psi^{(n)}$ will be continuous of compact support containing a neighborhood of the identity for some $n \geqq 2$. (8.2) implies that 


$$
\int f\left(g^{\prime} g\right) \psi^{(n)}\left(g^{\prime}\right) d g^{\prime}=\lambda^{n} f(g) .
$$

If $s(\cdot, x)$ satisfies $(8.2)$, then

$$
\int s(g, x) \psi^{(n)}(g) d g=\lambda^{n}
$$

If $s^{\prime} \sim s$ then $s^{\prime}(g, x)=(p(g x) / p(x)) s(g, x)$, so that if $s^{\prime}(\cdot, x) \in V_{\lambda}(\psi)$,

$$
\int p(g x) s(g, x) \psi^{(n)}(g) d g=\lambda^{n} p(x) .
$$

Then

$$
p(x)=\frac{\int p(g x) s(g, x) \psi^{(n)}(g) d g}{\int s(g, x) \psi^{(n)}(g) d g} .
$$

If $\Gamma \subset B(G)$ denotes the set where $p(x)$ attains its maximum, and $\psi^{(n)}(g)>0$, then $g \Gamma \subset \Gamma$, according to (8.4). Since this set of $g$ comprises a neighborhood of the identity, $G \Gamma \subset \Gamma$ so that $\Gamma=B(G)$. Hence $p$ is constant and $s^{\prime}=s$. This proves the lemma.

If the function $f$ on $G$ corresponds to the measure $\pi$, then equation (8.1) may be rewritten $\tilde{\mu} * \pi=\lambda \pi$. Here $\tilde{\mu}$ is the measure corresponding to the functions $\psi\left(g^{-1}\right)$. Because $\tilde{\mu}$ is absolutely continuous, $\pi$ must also be, so the set of positive solutions to $\tilde{\mu} * \pi=\lambda \pi$ is $V_{\lambda}(\psi)$. We also note that the non-negative solutions to (8.2) are all continuous and everywhere positive. This follows from (8.3) since $\psi^{(n)}$ is continuous, and if $f(g)=0$ then $f\left(g^{\prime} g\right)=0$ for $g^{\prime}$ in a neighborhood of the identity of $G$.

We may now apply Theorem 6.2 to the cone $V_{\lambda}(\psi)$. For, to begin with, this cone coincides with the cone of measures satisfying $\tilde{\mu} * \pi$ $=\lambda \pi$. Secondly, $\int f(g) \psi(g) d g=\lambda f(e)>0$ for every $f \in V_{\lambda}(\psi)$. So if $\varepsilon$ denotes the set of extremals of $V_{\lambda}(\psi)$ and $V_{\lambda}^{\prime}(\psi)$ denotes the set of functions in $V_{\lambda}(\psi)$ with $f(e)=1$ we have

Lemma 8.2. For every function $f \in V_{\lambda}(\psi)$ there is a unique measure $\nu$ on $V_{\lambda}^{\prime}(\psi) \cap \varepsilon$ with

$$
f(g)=\int_{V_{\lambda}^{\prime}(\psi) \cap \mathcal{E}} f_{\xi}(g) d \nu(\xi) .
$$

The deeper part of our analysis concerns the identification of the 
extremal functions in $V_{\lambda}^{\prime}(\psi)$ with multiplier functions. We shall first prove

Lemma 8.3. For each extremal $f \in V_{\lambda}^{\prime}(\psi) \cap \mathcal{E}$, there is a $G$-space $X$ on which $K$ is transitive, and a multiplier $s \in M(X)$, such that $f(g)=s(g, \xi)$ for some $\xi \in X$.

Proof. To begin with, let us notice that the space $V_{\lambda}^{\prime}(\psi) \cap \varepsilon$ is a $G$-space. For if $f(g)$ is an extremal of $V_{\lambda}^{\prime}(\psi)$, so is the function $f(g \gamma) / f(\gamma)$. Thus $G$ operates on $V_{\lambda}^{\prime}(\psi) \cap \mathcal{E}$ by right translation (and normalization). Let us form the subset $X$ of extremals $f(g \gamma) / f(\gamma), \gamma \in G$. If $L=\{\gamma: f(g \gamma)=c(\gamma) f(g)\}$, where $c(\gamma)$ is some arbitrary constant, then $X=G / L$. We can define a function $s$ on $G \times X$ by

$$
s(g, \gamma L)=f(g \gamma) / f(\gamma) .
$$

$s$ is well defined since if $\gamma$ is replaced by $\gamma l, l \in L$, both numerator and denominator in (8.6) are multiplied by $c(l)$. Moreover, $s$ is a multiplier in $M(X)$. To prove the lemma, it is merely necessary to show that $K$ is transitive on $X$. (This will, incidentally, imply that $X$ is compact, a fact which is not otherwise obvious.)

We shall use the following notation. If $\pi_{1}$ and $\pi_{2}$ are two measures we shall write $\pi_{1} \succ \pi_{2}$ if, for some $\epsilon>0, \pi_{1} \geqq \epsilon \pi_{2}$. We call a measure $\pi$ radial if it satisfies $k_{1} * \pi * k_{2}=\pi$ for $k_{1}, k_{2} \in K$.

If $\rho$ is any continuous function on $G$ with compact support, there is an $n$ with $\tilde{\psi}^{(n)}>\rho \cdot \tilde{\psi}$ is defined by $\tilde{\psi}(g)=\psi\left(g^{-1}\right)$. Choose $\rho$ radial and positive in a neighborhood of the identity. Then

$$
\tilde{\psi}^{(n)} * f=\lambda^{n} f,
$$

and so $\rho * f \prec f$. If we now choose $\rho>1$ on the support of $\tilde{\psi}$, we will also have $\tilde{\psi} * f \prec \rho * f$. Hence

$$
f \prec \rho * f \prec f .
$$

Since $\rho$ is radial, $m_{K} * \rho=\rho * m_{K}=\rho$. By Lemma 7.1,

$$
\rho * f * m_{K}=\rho * m_{K} * f * m_{K}=m_{K} * f * m_{K} * \rho=m_{K} * f * \rho .
$$

If $\rho^{\prime}$ is another radial function whose support contains a neighborhood of the identity, and $\rho^{\prime} \geqq 0$, we will have $\rho^{\prime} * \rho \succ \rho$. So

$$
\rho * f * \rho^{\prime}=\rho * m_{K} * f * \rho^{\prime}=m_{K} * f * \rho^{\prime} * \rho \succ m_{K} * f * \rho,
$$

or $\rho * f * \rho^{\prime} \succ \rho * f * m_{K}$. By (8.7),

$$
f * \rho^{\prime} \succ f * m_{K}
$$

Returning to the functional notation we have 


$$
\int_{G} f\left(g g^{\prime-1}\right) \rho^{\prime}\left(g^{\prime}\right) d g^{\prime} \succ \int_{K} f(g k) d k
$$

Setting $f(g)=s\left(g, \xi_{0}\right)$ (see (8.6)), we can write

$$
\int_{G} s\left(g, g^{\prime-1} \xi_{0}\right) s\left(g^{\prime-1}, \xi_{0}\right) \rho^{\prime}\left(g^{\prime}\right) d g^{\prime} \succ \int_{K} s\left(g, k \xi_{0}\right) s\left(k, \xi_{0}\right) d k
$$

or

$$
\int_{G} s\left(g, g^{\prime-1} \xi_{0}\right) \rho^{\prime}\left(g^{\prime}\right) d g^{\prime} \succ \int_{K} s\left(g, k \xi_{0}\right) d k
$$

which may be written

$$
\int_{X} s(g, \xi) d \omega_{1}(\xi) \succ \int_{X} s(g, \xi) d \omega_{2}(\xi)
$$

Now the functions $s(g, \xi)$ are extremals and $V_{\lambda}(\psi)$ is a lattice. By the corollary to Theorem $6.1,(8.11)$ implies that $\omega_{1} \succ \omega_{2}$. Now $X$ is a manifold as the quotient of a Lie group by a closed subgroup, and the measure $\omega_{1}$, as image under the natural map of $G \rightarrow G / L$ of an absolutely continuous measure, is itself absolutely continuous on $X$. (This means that the restriction to a coordinate neighborhood is absolutely continuous with respect to Lebesgue measure induced on that coordinate neighborhood.) On the other hand, $\omega_{2}=m_{K} * \xi_{0}$ is concentrated on the submanifold $K \xi_{0} \subset X$. A measure on a submanifold of $X$ can be absolutely continuous on $X$ only if the submanifold is open. But $K \xi_{0}$ is closed and $X$ is connected. It follows that $K \xi_{0}=X$ which is what was needed to prove the lemma.

To prove that the space $X$ occurring in Lemma 8.3 is $B(G)$ or an equivariant image of $B(G)$, we use the next lemma. We shall continue to use the notation $\pi_{1} \succ \pi_{2}$ introduced in the proof of Lemma 8.3.

LEMMA 8.4. Let $Y$ and $Z$ be two homogeneous $K$-spaces and suppose there is a map from $Y$ to the space of probability measures on $Z, y \rightarrow \theta_{y}$, such that $\theta_{k y}=k \theta_{y}$ for $k \in K$, and whenever $\mu_{1}, \mu_{2}$ are two positive measures on $K$ such that $\mu_{1} * \theta_{y} \succ \mu_{2} * \theta_{y}$, we have $\mu_{1} * y \succ \mu_{2} * y$. Then there is a map $j: Z \rightarrow Y$, equivariant with respect to $K$, and such that $j^{-1}(y)$ coincides with the support of $\theta_{y}$ for each $y \in Y$.

Proof. We set $Y=K / K_{1}, Z=K / K_{2}$. If $m_{K_{1}}$ denotes the Haar measure on $K_{1}$, then $Y$ may be identified with the space of measures $\left\{k m_{K_{1}}: k \in K\right\}$ on $K$. Similarly, the space of measures on $Z$ can be identified with the space of measures $\left\{\nu * m_{K_{2}}: \nu\right.$ a measure on $\left.K\right\}$. Namely, if $\omega$ is a measure on $Z$, set 


$$
\bar{\omega}(F)=\int_{K_{/ K_{2}}}\left[\int_{K_{2}} F\left(k k^{\prime}\right) d k^{\prime}\right] d \omega\left(k K_{2}\right),
$$

so that $\tilde{\omega}$ is a measure on $K$. Then $\tilde{\omega} k=\tilde{\omega}$ for $k \in K_{2}$, and so $\tilde{\omega}=\tilde{\omega} * m_{K_{2}}$. Since $\tilde{\omega} \rightarrow \omega$ under the natural map of $K \rightarrow Z$, the correspondence $\tilde{\omega} \leftrightarrow \omega$ is $1-1$.

Let $y_{0} \in Y$ correspond to the coset $K_{1}$ or to the measure $m_{K_{1}}$. If $\theta_{\nu_{0}}$ corresponds to $\nu * m_{K_{2}}$, then, since $K_{1}$ leaves $y_{0}$ fixed, it must leave $\theta_{y_{0}}$ fixed, so that $\theta_{y_{0}}=m_{K_{1}} * \theta_{\nu_{0}}$, and $\theta_{\nu_{0}}$ corresponds to $m_{K_{1}} * \nu * m_{K_{2}}$. The map $y \rightarrow \theta_{y}$ is then given by

$$
k m_{K_{1}} \rightarrow k m_{K_{1}} * \nu * m_{K_{2}} .
$$

The hypothesis of the lemma may then be rewritten: $\mu_{1} * m_{K_{1}} * \nu * m_{K_{2}}$ $\succ \mu_{2} * m_{K_{1}} * \nu * m_{K_{2}}$ implies $\mu_{1} * m_{K_{1}} \succ \mu_{2} * m_{K_{1}}$. We can make this more precise. Suppose, in fact, that $\mu_{1} * m_{K_{1}} * \nu * m_{K_{2}} \geqq \mu_{2} * m_{K_{1}} * \nu * m_{K_{2}}$. Then for some $C>0, \mu_{1} * m_{K_{1}} \geqq C \mu_{2} * m_{K_{1}}$. Take the largest possible $C$ for which this is true and suppose $C<1$. Then

$$
\left(\mu_{1} * m_{K}-C \mu_{2} * m_{K_{1}}\right) * m_{K_{1}} * \nu * m_{K_{2}} \geqq(1-C) \mu_{2} * m_{K_{1}} * \nu * m_{K_{2}} .
$$

Now $\mu_{1} * m_{K}-C \mu_{2} * m_{K_{1}}$ is a positive measure, so we can apply the condition in the lemma again. We get, for some $\epsilon>0$,

$$
\left(\mu_{1} * m_{K_{1}}-C \mu_{2} * m_{K_{1}}\right) * m_{K_{1}} \geqq \epsilon \mu_{2} * m_{K_{1}} \text {, }
$$

or

$$
\mu_{1} * m_{K_{1}} \geqq(C+\epsilon) \mu_{2} * m_{K_{1}} \text {. }
$$

This contradicts the choice of $C$. It follows that $C \geqq 1$. What we have shown is that, for a signed measure $\mu$ on $K$,

$$
\mu * m_{K_{1}} * \nu * m_{K_{2}} \geqq 0 \text { implies } \mu * m_{K_{1}} \geqq 0 \text {. }
$$

Suppose, more generally, that we have two positive measures $\omega_{1}$ and $\omega_{2}$ on $K$ with the property that $\mu * \omega_{1} \geqq 0$ implies $\mu * \omega_{2} \geqq 0$. We claim that we must have $\omega_{2}=\omega_{1} * \omega$ for some positive measure $\omega$ on $K$. The proof is based on the Hahn-Banach theorem. Namely, form the cone of all $\omega_{1} * \omega, \omega \geqq 0$. This cone is weakly closed. If $\omega_{2}$ did not belong to this cone, it could be separated from it by a continuous linear functional. That is, there would exist a continuous function $h$ with $\omega_{1} * \omega(h) \geqq 0$ for all $\omega \geqq 0$ on $K$, but $\omega_{2}(h)<0$. Now the first inequality implies that $\tilde{h} * \omega_{1} \geqq 0\left(\tilde{h}(g)=h\left(g^{-1}\right)\right)$. Hence $h * \omega_{2} \geqq 0$ which in turn implies $\omega_{2}(h) \geqq 0$. So $\omega_{2}$ must belong to the cone in question, or $\omega_{2}=\omega_{1} * \omega$.

By (8.13) we therefore have $m_{K_{1}}=m_{K_{1}} * \nu * m_{K_{2}} * \omega$ for a positive measure $\omega$ on $K$. Let $P$ be the support of $\nu$ and $Q$ the support of $\omega$. 
The support of a convolution of positive measures is the product of the supports, so $P K_{2} Q \subset K_{1}$. This gives $P K_{2} P^{-1} \subset P K_{2} Q Q^{-1} K_{2} P^{-1} \subset K_{1}$.

To define the map $j$ it is merely necessary to verify that every point of $Z$ belongs to the support of some $\theta_{v}$ and that the supports of $\theta_{v_{1}}$ and $\theta_{y_{2}}$ do not overlap unless $y_{1}=y_{2}$. As to the first question, we notice that since some point belongs to the support of some $\theta_{y}$, and $Z$ is homogeneous, every point belongs to the support of some $\theta_{y}$. For the second question we observe that the support of $\theta_{k K_{1}}$ is the image in $Z$ of $k K_{1} P K_{2}$ by (8.12). So if

$$
k^{\prime} K_{1} P K_{2} \cap k^{\prime \prime} K_{1} P K_{2} \neq \varnothing,
$$

then $k^{\prime-1} k^{\prime \prime} \in K_{1} P K_{2} P^{-1} K_{1}^{-1} \subset K_{1}$ and $k^{\prime} K_{1}=k^{\prime \prime} K_{1}$. This completes the proof of the lemma.

We return to the extremal $f$ in $V_{\lambda}^{\prime}(\psi)$ and the space $X$ which it generates. We have $f=s\left(\cdot, \xi_{0}\right), s \in M(X)$, and we have shown that $K$ is transitive on $X$. At this point we invoke the theory of semi-spherical functions. By Lemma 2.1, every multiplier in $M(X)$ is equivalent to a $K$-multiplier:

$$
s(g, \xi)=\frac{p(g \xi)}{p(\xi)} \sigma(g, \xi), \quad \sigma \in M_{K}(X),
$$

where $p(\xi)$ is continuous and positive in $X$. By Lemma 5.1 , the functions $\sigma(\cdot, \xi)$ are semi-spherical and belong to the spherical function

$$
\phi(g)=\int_{X} \sigma(g, \xi) d m(\xi),
$$

where $m$ is the unique $K$-invariant measure on $X$. By Theorem 7.1, the semi-spherical functions belonging to $\phi$ are all generated by some $\sigma_{\phi} \in M_{K}(B(G)):$

$$
\sigma(g, \xi)=\int_{B(G)} \sigma_{\phi}(g, x) d \theta_{\xi}^{\prime}(x),
$$

for some measure $\theta^{\prime}$ on $B(G)$. Let $B^{\prime}$ be the equivariant image of $B(G)$ obtained by identifying $x_{1}, x_{2} \in B(G)$ if $\sigma_{\phi}\left(g, x_{1}\right)=\sigma_{\phi}\left(g, x_{2}\right)$. (One verifies readily that this is an equivalence relationship.) $\sigma_{\phi}$ defines a multiplier $\sigma_{\phi}^{\prime}$ in $M_{K}\left(B^{\prime}\right)$ and we may write

$$
\sigma(g, \xi)=\int_{B^{\prime}} \sigma_{\phi}^{\prime}(g, y) d \theta_{\xi}(y) \text {. }
$$

Because the $\sigma_{\phi}^{\prime}(\cdot, y), y \in B^{\prime}$, are all distinct, the points of $B^{\prime}$ correspond to the normalized extremals of $V_{\phi}$. In particular, the meas- 
ures $\theta_{\xi}$ in (8.15) are uniquely determined. Since $\sigma(g, k \xi)=\sigma(g k, \xi)$,we conclude from (8.15) that $k \theta_{\xi}=\theta_{k \xi}$. We now show that the map $\xi \rightarrow \theta_{\xi}$ from $X$ to the probability measures on $B^{\prime}$ satisfies the condition of Lemma 8.4. Suppose then that $\mu_{1}, \mu_{2}$ are positive measures on $K$ with $\mu_{1} * \theta_{\xi} \succ \mu_{2} * \theta_{\xi}$ for some $\xi \in X$. Note that by (8.14), $s \succ \sigma \succ s$. As functions of $g$ we then have

$$
\begin{aligned}
& \int_{K} s(g, k \xi) d \mu_{1}(k) \succ \int_{K} \sigma(g, k \xi) d \mu_{1}(k) \\
& =\int_{K} \sigma(g k, \xi) d \mu_{1}(k)=\int_{B^{\prime}} \int_{K} \sigma_{\phi}^{\prime}(g k, y) d \mu_{1}(k) d \theta_{\xi}(y) \\
& =\int_{B^{\prime}} \int_{K} \sigma_{\phi}^{\prime}(g, k y) d \mu_{1}(k) d \theta_{\xi}(y)=\int_{B^{\prime}} \sigma_{\phi}^{\prime}(g, z) d \mu_{1} * \theta_{\xi}(z) \\
& \quad \succ \int_{B^{\prime}} \sigma_{\phi}^{\prime}(g, z) d \mu_{2} * \theta_{\xi}(z)=\int_{B^{\prime}} \int_{K} \sigma_{\phi}^{\prime}(g, k y) d \mu_{2}(k) d \theta_{\xi}(y) \\
& =\int_{B^{\prime}} \int_{K} \sigma_{\phi}^{\prime}(g k, y) d \mu_{2}(k) d \theta_{\xi}(y)=\int_{K} \sigma(g k, \xi) d \mu_{2}(k) \\
& =\int_{K} \sigma(g, k \xi) d \mu_{2}(k) \succ \int s(g, k \xi) d \mu_{2}(k) .
\end{aligned}
$$

Now the extreme ends of this series of inequalities are integrals over $s(g, \eta), \eta \in X$, and these are extremals in $V_{\lambda}^{\prime}(\psi)$. By the corollary to Theorem 6.1 , since $V_{\lambda}(\psi)$ is a lattice, we must have $\mu_{1} * \xi \succ \mu_{2} * \xi$. The conditions of Lemma 8.4 are thereby verified and we can conclude that there is a map $j: B^{\prime} \rightarrow X$ such that $\theta_{\xi}$ has its support equal to $j^{-1}(\xi)$.

Finally we must show that $j$ is equivariant as a map of $G$-spaces: $j(g y)=g j(y)$. By (8.15),

$$
\begin{aligned}
\sigma(g \gamma, \xi) & =\sigma(g, \gamma \xi) \sigma(\gamma, \xi) \\
& =\sigma(\gamma, \xi) \int_{B^{\prime}} \sigma_{\phi}^{\prime}(g, z) d \theta_{\gamma \xi}(z) .
\end{aligned}
$$

On the other hand,

$$
\begin{aligned}
\sigma(g \gamma, \xi) & =\int_{B^{\prime}} \sigma_{\phi}^{\prime}(g \gamma, y) d \theta_{\xi}(y)=\int_{B^{\prime}} \sigma_{\phi}^{\prime}(g, \gamma g) \sigma_{\phi}^{\prime}(\gamma, y) d \theta_{\xi}(g) \\
& =\int \sigma_{\phi}^{\prime}(g, z) \sigma_{\phi}^{\prime}\left(\gamma, \gamma^{-1} z\right) d \gamma \theta_{\xi}(z) .
\end{aligned}
$$


By the uniqueness of the Choquet-Deny representation in $V_{\phi}$ we must have

$$
\sigma(\gamma, \xi) d \theta_{\gamma \xi}(z)=\sigma_{\phi}^{\prime}\left(\gamma, \gamma^{\prime} z\right) d \gamma \theta_{\xi}(z) .
$$

This implies that

$$
\gamma \theta_{\xi} \prec \theta_{\gamma \xi}
$$

for any $\gamma \in G$. Suppose now that $j(y)=\xi$. Then $y \in \operatorname{support}\left(\theta_{\xi}\right)$ and $g y \in \operatorname{support}\left(g \theta_{\xi}\right)$. By (8.18) this implies that $g y \in \operatorname{support}\left(\theta_{g \xi}\right)$ or $j(g \xi)=g y$. This proves that $j$ is an equivariant map of $B^{\prime}$ onto $X$. Now $B^{\prime}$, in turn, is an equivariant image of $B(G)$. But the map $B(G) \rightarrow X$ induces an injection of $M(X) \rightarrow M(B(G))$ and so the multiplier $s \in M(X)$ corresponds to a multiplier $s^{*} \in M(B(G))$. We have proved

THEOREM 8.1. The cone $V_{\lambda}(\psi)$ of non-negative solutions to

$$
\int f\left(g^{\prime} g\right) \psi\left(g^{\prime}\right) d g^{\prime}=\lambda f(g)
$$

admits a Choquet-Deny representation in terms of its normalized extremals. Each normalized extremal has the form $s(\cdot, x)$, where $s \in M(B(G)$ ) and $x \in B(G)$. Moreover, if $\eta \in H(B(G))$, there is at most one multiplier $s_{\eta} \in \eta$ such that $s_{\eta}(\cdot, x) \in V_{\lambda}(\psi)$.

9. Basic multipliers. Theorem 8.1 tells us that the extremals of $V_{\lambda}(\psi)$ are proportional to multiplier functions in $E(B(G))$. However, not all the multiplier functions in $V_{\lambda}(\psi)$ need be extremals. For example, taking $\phi \equiv 1$ on $G$, the multipliers $\left(d g^{-1} m / d m\right)(x)=\sigma_{1}(g, x)$ and $\sigma_{0}(g, x) \equiv 1$ in $M_{K}(B(G))$ both belong to $V_{\phi}$. However,

$$
1=\int_{B(G)} \frac{d g^{-1} m}{d m}(x) d m(x),
$$

so that $\sigma_{0}$ cannot be an extremal of $V_{\phi}$. In this section we shall introduce a class of multipliers, the basic multipliers, such that the normalized extremals of $V_{\lambda}(\psi)$ coincide exactly with the set of basic multiplier functions in $V_{\lambda}(\psi)$. In the course of this discussion we shall resolve another question. Namely we shall show that the space $X$ of the preceding section is not just an image of $B(G)$ but coincides with $B(G)$. In addition we will find that the space $B^{\prime}=B(G)$ so that the space $B(G)$ coincides with the set of extremals in each $V_{\phi}$.

Definition 9.1. $s \in M(B(G))$ is basic if, for a signed measure $\omega$ on $B(G)$, 


$$
\int s(g, x) d \omega(x) \geqq 0 \text { for all } g \text { implies } \omega \geqq 0 \text {. }
$$

We denote by $M_{b}(B(G))$ the set of all basic multipliers, and by $E_{b}(B(G))$ the set of all multiplier functions coming from basic multipliers.

In particular, $\int s(g, x) d \omega(x)=0$ implies $\omega=0$ for $s$ basic, and so the functions $s(\cdot, x), x \in B(G)$, must all be distinct. It follows that a multiplier on $B(G)$ induced by a multiplier in an equivariant image $B^{\prime}$ of $B(G)$ cannot be basic unless $B^{\prime}=B(G)$.

LEMMA 9.1. $s \in M(B(G))$ is basic if and only if

$$
\int s(g, x) d \omega_{1}(x) \succ \int s(g, x) d \omega_{2}(x)
$$

implies $\omega_{1} \succ \omega_{2}$ for any two positive measures $\omega_{1}, \omega_{2}$ on $B(G)$.

The proof is similar to the proof of (8.13) in Lemma 8.4.

LEMMA 9.2. If $s_{1}$ and $s_{2}$ are equivalent multipliers then $s_{1}$ is basic if and only if $s_{2}$ is basic.

Proof. For, if $s_{1}$ and $s_{2}$ are equivalent, $s_{1} \succ s_{2} \succ s_{1}$, so that (9.1) for $s_{1}$ is equivalent to the same assertion for $s_{2}$.

Thus the condition of being basic depends only on the equivalence class of $s$ and we may introduce the set $H_{b}(B(G)$ ) of basic (cohomology) classes.

LEMMA 9.3. $s$ is basic if and only if the cone of functions on $B(G)$ spanned by the functions $s(\mathrm{~g}, \cdot)$ is dense in the cone of all positive continuous functions on $B(G)$.

That $s$ is basic under the stated condition is immediate. The converse is a consequence of the Hahn-Banach theorem.

According to Lemma 9.2, $s$ is basic if and only if the $K$-multiplier equivalent to $s$ is basic. We will now show that the basic $K$-multipliers are just the $\sigma_{\phi}$ which we saw spanned the cones $V_{\phi}$.

THEOREM 9.1. $\sigma \in M_{K}(B(G))$ is basic if and only if $\sigma=\sigma_{\phi}$ for some spherical function $\phi$.

Proof. We shall first show that if $\sigma$ is basic it coincides with some $\sigma_{\phi}$. This is the easier of the two directions. Suppose then that $\sigma$ is basic. In any case $\sigma \in V_{\phi}$ for some spherical function $\phi$ by Lemma 5.1. Then, as in the proof of Theorem 8.1, 


$$
\sigma(g, x)=\int_{B^{\prime}} \sigma_{\phi}^{\prime}(g, y) d \theta_{x}(y) .
$$

Now suppose that $\mu_{1} * \theta_{\xi} \succ \mu_{2} * \theta_{\xi}$ where $\mu_{1}, \mu_{2}$ are measures on $K$. Then

$$
\begin{aligned}
& \int_{B} \sigma(g, y) d \mu_{i} * x(y) \\
& \quad=\int_{K} \sigma(g, k x) d \mu_{i}(k)=\int_{K} \sigma(g k, x) d \mu_{i}(k) \\
& \quad=\int_{K} \int_{B^{\prime}} \sigma_{\phi}^{\prime}(g k, z) d \theta_{x}(z) d \mu_{i}(k)=\int_{K} \int_{B^{\prime}} \sigma_{\phi}^{\prime}(g, k z) d \theta_{x}(z) d \mu_{i}(k) \\
& =\int_{B^{\prime}} \sigma_{\phi}^{\prime}(g, u) d \mu_{i} * \theta_{x}(u),
\end{aligned}
$$

for $i=1$, 2. Since $\mu_{1} * \theta_{x} \succ \mu_{2} * \theta_{x}$ it follows that

$$
\int \sigma(g, y) d \mu_{1} * x(y) \succ \int \sigma(g, y) d \mu_{2} * x(y) .
$$

By Lemma 9.1, since $\sigma$ is basic, this implies $\mu_{1} * x \succ \mu_{2} * x$. We see that the map $x \rightarrow \theta_{x}$ from $B(G)$ to $B^{\prime}$ fulfills the conditions of Lemma 8.4. This means that there is an equivariant map (see the proof of Theorem 8.1) $j: B^{\prime} \rightarrow B(G)$ such that $\theta_{x}$ has its support on $j^{-1}(x)$. But $B^{\prime}$ is itself an equivariant image of $B(G)-j^{\prime}: B(G) \rightarrow B^{\prime}$, so that $j j^{\prime}$ is an equivariant map of $B(G)$ onto itself. Now, by Lemma 7.2 , the identity is the only equivariant map of $B(G)$ onto itself. So $j j^{\prime}$ is the identity. Since $j^{\prime}$ is onto, $j$ is an isomorphism. Hence $B^{\prime} \approx B(G)$ and $j$ is an equivariant map of $B(G)$ onto itself; hence the identity. Since $\theta_{x}$ has its support on $j^{-1}(x)$, it follows that $\theta_{x}=\delta_{x}$ and $\sigma(g, x)$ $=\sigma_{\phi}^{\prime}(g, x)=\sigma_{\phi}(g, x)$. This proves that if $\sigma$ is basic, then $\sigma=\sigma_{\phi}$.

Now consider the $K$-multiplier $\sigma_{\phi}$ for some spherical $\phi$. We wish to prove that $\sigma_{\phi}$ is basic. We must first establish that $\sigma_{\phi}$ separates points in $B(G)$. To do this introduce the space $B^{\prime}$ as before: $B^{\prime}$ is obtained from $B(G)$ by identifying $x_{1}, x_{2} \in B(G)$ if $\sigma_{\phi}\left(g, x_{1}\right)=\sigma_{\phi}\left(g, x_{2}\right)$ for all $g \in G$. Let $j$ denote the ensuing natural map of $B(G) \rightarrow B^{\prime}$. We then define $\sigma_{\phi}^{\prime}$ as before by $\sigma_{\phi}^{\prime}(g, j(x))=\sigma_{\phi}(g, x)$.

For each $\xi \in B^{\prime}$, let $K_{\xi} \subset K$ denote the subgroup of $K$ that leaves $\xi$ fixed. $K_{\xi}$ takes $j^{-1}(\xi)$ into itself and is transitive on $j^{-1}(\xi)$ since $K$ is transitive on $B(G)$. So there is a unique probability measure $\theta_{\xi}$ on $j^{-1}(\xi)$ invariant under $K_{\xi}$. We see readily that $k \theta_{\xi}=\theta_{k \xi}$ for $k \in K$. If 
$\gamma \in G$ and $\gamma \xi=\xi$, then $\gamma$ determines a differentiable homeomorphism of $j^{-1}(\xi)$ with itself and so $\gamma \theta_{\xi}$ is absolutely continuous with respect to $\theta_{\xi}$. Since every $g \in G$ is a product $k \gamma$ with $k \in K, \gamma \xi=\xi$ (since $K \xi=B^{\prime}$ ), it follows that $g \theta_{\xi}=k \gamma \theta_{\xi}$ is absolutely continuous with respect to $k \theta_{\xi}=\theta_{k \xi}=\theta_{k \gamma \xi}=\theta_{g \xi}$. Then $g^{-1} \theta_{g \xi}$ is absolutely continuous with respect to $\theta_{\xi}$ and the expression

$$
\sigma(g, x)=\frac{d g^{-1} \theta_{o \xi}}{d \theta_{\xi}}(x) \sigma_{\phi}^{\prime}(g, \xi)
$$

is well defined for $x$ in the support of $\theta_{\xi}$, that is, for $j(x)=\xi$. We claim that with this definition of $\xi, \sigma(g, x)$ defines a multiplier in $M_{K}(B(G))$. $\sigma(k, x)=1$ since $k^{-1} \theta_{k \xi}=\theta_{\xi}$ and $\sigma_{\phi}^{\prime}(k, \xi)=1$. What remains to be verified is the multiplier identity. Letting $\xi=j(x)$ we have, making use of (3.13):

$$
\begin{aligned}
\sigma\left(g_{1} g_{2}, x\right) & =\frac{d g_{2}^{-1} g_{1}^{-1} \theta_{\theta_{1} g_{2} \xi}}{d \theta_{\xi}}(x) \sigma_{\phi}^{\prime}\left(g_{1} g_{2}, \xi\right) \\
& =\frac{d g_{1}^{-1} \theta_{o_{1} 0_{2} \xi}}{d g_{2} \theta_{\xi}}\left(g_{2} x\right) \sigma_{\phi}^{\prime}\left(g_{1}, g_{2} \xi\right) \sigma_{\phi}^{\prime}\left(g_{2}, \xi\right) \\
& =\frac{d g_{1}^{-1} \theta_{\sigma_{10} \xi}}{d \theta_{\sigma_{2} \xi}}\left(g_{2} x\right) \frac{d \theta_{\sigma_{2} \xi}}{d g_{2} \theta_{\xi}}\left(g_{2} x\right) \sigma_{\phi}^{\prime}\left(g_{1}, g_{2} \xi\right) \sigma_{\phi}^{\prime}\left(g_{2}, \xi\right) \\
& =\sigma\left(g_{1}, g_{2} x\right) \frac{d g_{2}^{-1} \theta_{g_{2} \xi}}{d \theta_{\xi}}(x) \sigma_{\phi}^{\prime}\left(g_{2}, \xi\right) \\
& =\sigma\left(g_{1}, g_{2} x\right) \sigma\left(g_{2}, x\right) .
\end{aligned}
$$

Here we have used the fact that $j\left(g_{2} x\right)=g_{2} \xi$ if $j(x)=\xi$.

We next verify that this multiplier $\sigma$ also belongs to $\phi$. It suffices to show that

$$
\int_{B(G)} \sigma(g, x) d m(x)=\phi(g) .
$$

But

$$
\begin{aligned}
\int_{B(G)} \sigma(g, x) d m(x) & =\int_{K} \sigma\left(g, k x_{0}\right) d k \\
& =\int_{K} \int_{K^{\prime}} \sigma\left(g, k k^{\prime} x_{0}\right) d k d k^{\prime} .
\end{aligned}
$$

Here $x_{0}$ is any point of $B(G)$ and $K^{\prime}$ is any subgroup of $K$. If we let 
$\xi_{0}=j\left(x_{0}\right)$ and set $K^{\prime}=K_{\xi_{0}}$, then $k^{\prime} x_{0}$ has the distribution $\theta_{\xi_{0}}$ for $k^{\prime}$ uniformly distributed in $K^{\prime}$. So

$$
\begin{aligned}
\int_{B(G)} \sigma(g, x) d m(x) & =\int_{K} \int_{B(G)} \sigma(g, k y) d \theta_{\xi_{0}}(y) d k \\
& =\int_{K} \int_{B(G)} \sigma(g k, y) d \theta_{\xi_{0}}(y) d k .
\end{aligned}
$$

Now

$$
\begin{aligned}
\int_{B(G)} \sigma(g, y) d \theta_{\xi_{0}}(y) & =\int_{j^{-1}\left(\xi_{0}\right)} \sigma(g, y) d \theta_{\xi_{0}}(y) \\
& =\int_{j^{-1}\left(\xi_{0}\right)} \frac{d g^{-1} \theta_{o \xi_{0}}}{d \theta_{\xi_{0}}}(y) \sigma_{\phi}^{\prime}\left(g, \xi_{0}\right) d \theta_{\xi_{0}}(y) \\
& =\sigma_{\phi}^{\prime}\left(g, \xi_{0}\right) \int d g^{-1} \theta_{o \xi_{0}}(y)=\sigma_{\phi}^{\prime}\left(g, \xi_{0}\right) .
\end{aligned}
$$

By (9.5),

$$
\begin{aligned}
\int_{B(G)} \sigma(g, x) d m(x) & =\int_{\boldsymbol{K}} \sigma_{\phi}^{\prime}\left(g k, \xi_{0}\right) d k=\int_{\boldsymbol{K}} \sigma_{\phi}\left(g k, x_{0}\right) d k \\
& =\int_{\boldsymbol{K}} \sigma_{\phi}\left(g, k x_{0}\right) d k=\int_{B(G)} \sigma_{\phi}(g, x) d m(x)=\phi(g) .
\end{aligned}
$$

This proves that $\sigma$ belongs to $\phi$. But (9.6) also shows that $\sigma_{\phi}\left(g, x_{0}\right)$ $=\sigma_{\phi}^{\prime}\left(g, \xi_{0}\right)$ is expressed as a linear combination of functions in $V_{\phi}$. Since the $\sigma_{\phi}$ are extremals this can only take place if all the $\sigma(g, y)$ for $y \in j^{-1}\left(\xi_{0}\right)$ coincide with $\sigma_{\phi}\left(g, x_{0}\right)$. In other words, $d g^{-1} \theta_{g \xi_{0}} / d \theta_{\xi_{0}}$ must be identically 1 , or, equivalently, the measure $g^{-1} \theta_{g \xi_{0}}$ coincides with $\theta_{\xi_{0}}$. The same argument implies that for all $g \in G, \xi \in B^{\prime}, g \theta_{\xi}=\theta_{g \xi}$. But $B(G)$ is a boundary (see $\S 3$ ) so that some $g_{n} \theta_{\xi}$ tends to a point measure. Setting $g_{n}=k_{n} \gamma_{n}$, where $\gamma_{n} \xi=\xi$ and $k_{n} \in K$, we must have $\gamma_{n} \theta_{\xi}=\theta_{\gamma_{n} \xi}=\theta_{\xi}$ tending to a point measure. This means that $\theta_{\xi}$ is a point measure and so $j$ is $1-1$. We have thereby proved that $\sigma_{\phi}(g, x)$ separates points in $B(G)$.

It is now an easy matter to show that $\sigma_{\phi}$ is basic. For, since the functions $\sigma_{\phi}(\cdot, x)$ are distinct for distinct $x \in B(G)$, it follows that $B(G)$ coincides with the normalized extremals of $V_{\phi}$. Now suppose $\int_{B(G)} \sigma_{\phi}(g, x) d \omega(x) \geqq 0$ for some signed measure $\omega$ on $B(G)$. From the fact that $V_{\phi}$ is a lattice, by the corollary to Theorem 6.1, this inequal- 
ity implies that $\omega \geqq 0$. This, however, was just the condition for $\sigma_{\phi}$ to be basic. This completes the proof of the theorem.

Corollary. A multiplier $s$ is basic if and only if it is equivalent to $\sigma_{\phi}$ for some spherical function $\phi$.

Very little is known regarding the subset $H_{b}(B(G))$ of the linear space $H(B(G))$. For example, is it a semigroup, and is it closed under the operations $\sigma \rightarrow \sigma^{r}, r \geqq 1$ ?

10. Application to the Choquet-Deny representation. We shall apply the results of $\S 9$ to obtain more precise information regarding the extremals of $V_{\lambda}(\psi)$.

THEOREM 10.1. If $s(\cdot, x)$ is an extremal of $V_{\lambda}(\psi)$, then the multiplier $s$ is basic.

Proof. As in the proof of Theorem 9.1, we form the space $B^{\prime}$ which is obtained from $B(G)$ by identifying $x_{1}, x_{2} \in B(G)$ if $s\left(g, x_{1}\right)$ $=s\left(g, x_{2}\right)$. We have an equivariant map $j: B(G) \rightarrow B^{\prime}$, and $s(g, x)$ $=s^{\prime}(g, j(x))$, where $s^{\prime} \in M\left(B^{\prime}\right)$. Again, it will suffice to prove that $B^{\prime}=B(G)$. For, $V_{\lambda}(\psi)$ is a lattice, and so by the corollary to Theorem 6.1 , if $\int s^{\prime}(g, \xi) d \omega(\xi) \geqq 0$, then $\omega \geqq 0$.

Let $\sigma^{\prime}$ be the $K$-multiplier equivalent to $s^{\prime}$ on $B^{\prime}$. The functions $\sigma^{\prime}(\cdot, \xi)$ are semi-spherical and belong to $V_{\phi}$ for some $\phi$. So, by Theorem 7.1,

$$
\sigma^{\prime}(g, \xi)=\int_{B(G)} \sigma_{\phi}(g, x) d \theta_{\xi}(x),
$$

where $\sigma_{\phi}$ is the basic multiplier belonging to $\phi$. Because $\sigma_{\phi}$ is basic, the measures $\theta_{\xi}$ in (10.1) are uniquely determined. Now use the multiplier equation:

$$
\begin{aligned}
\sigma^{\prime}(\gamma g, \xi) & =\int \sigma_{\phi}(\gamma g, x) d \theta_{\xi}(x) \\
& =\int \sigma_{\phi}(\gamma, g x) \sigma_{\phi}(g, x) d \theta_{\xi}(x)=\int \sigma_{\phi}(\gamma, x) \sigma_{\phi}\left(g, g^{-1} x\right) d g \theta_{\xi}(x)
\end{aligned}
$$

But $\sigma^{\prime}(\gamma g, \xi)$ is also equal to

$$
\sigma^{\prime}(\gamma, g \xi) \sigma^{\prime}(g, \xi)=\int \sigma_{\phi}(\gamma, x) \sigma^{\prime}(g, \xi) d \theta_{o \xi}(x) .
$$


Comparing the two expressions as functions of $\gamma$, we have

$$
\sigma_{\phi}\left(g, g^{-1} x\right) d g \theta_{\xi}(x)=\sigma^{\prime}(g, \xi) d \theta_{\sigma \xi}(x) \text {. }
$$

Now we can write

$$
s^{\prime}(g, \xi)=\frac{p^{\prime}(g \xi)}{p(\xi)} \sigma^{\prime}(g, \xi),
$$

where $p^{\prime}$ is a positive continuous function on $B^{\prime}$. Since $s^{\prime}(\cdot, \xi) \in V_{\lambda}(\psi)$,

$$
\int p^{\prime}(g \xi) \sigma^{\prime}(g, \xi) \psi(g) d g=\lambda p^{\prime}(\xi)
$$

Form the convex set $Q$ of all functions $p(x)$, continuous and nonnegative on $B(G)$, and satisfying

$$
\int p(x) d \theta_{\xi}(x)=p^{\prime}(\xi)
$$

If we recall the proof of Theorem 8.1 we see that the maps $\xi \rightarrow \theta_{\xi}$ of (8.15) and (10.1) are the same. As a result $\theta_{\xi}$ has its support on $j^{-1}(\xi)$. It follows that $Q$ is nonempty since the function $p=p^{\prime} \circ j^{-1}$ will belong to $Q$.

Define a linear transformation $T$ on the space of continuous functions on $B(G)$ by

$$
T p(x)=\lambda^{-1} \int p(g x) \sigma_{\phi}(g, x) \psi(g) d g .
$$

Then

$\lambda^{-1} \iint p(g x) \sigma_{\phi}(g, x) \psi(g) d g d \theta_{\xi}(x)=\lambda^{-1} \iint p(x) \sigma_{\phi}\left(g, g^{-1} x\right) d g \theta_{\xi}(x) \psi(g) d g$.

By (10.2), we have

(10.5) $\int T p(x) d \theta_{\xi}(x)=\lambda^{-1} \iint p(x) \sigma^{\prime}(g, \xi) d \theta_{o \xi}(x) \psi(g) d g$.

If $p \in Q$, this equals

$$
\lambda^{-1} \int p^{\prime}(g \xi) \sigma^{\prime}(g, \xi) \psi(g) d g=p^{\prime}(\xi) .
$$

It follows that $T$ takes $Q$ into itself.

The functions in $T Q$ form an equicontinuous family. For 


$$
\begin{aligned}
\mid T p(k x)- & T p(x) \mid \\
& \leqq \lambda^{-1} \int p(g x) \sigma_{\phi}(g, x)\left(\left|\psi\left(k^{-1} g\right)-\psi(g)\right|\right) d g . \\
& =\lambda^{-1} \iint p\left(k_{1} g x\right) \sigma_{\phi}(g, x)\left(\left|\psi\left(k^{-1} k_{1} g\right)-\psi\left(k_{1} g\right)\right|\right) d g d k_{1} \\
& \leqq A \int p(y) d m(y) \cdot \max _{g}\left|\psi\left(k^{-1} g\right)-\psi(g)\right|,
\end{aligned}
$$

where $A=\lambda^{-1} \max \sigma_{\phi}(g, x)$ and the maximum is taken over all $x \in B(G)$ and all $g$ for which some $k^{\prime} g$ is in the support of $\psi, k^{\prime} \in K$. Now, by (10.4),

$$
\int p(y) d m(y)=\int p^{\prime}(\xi) d m(\xi)
$$

so that the right-hand side of (10.6) goes to 0 as $k x \rightarrow x$ independently of $p \in Q$.

As a result, $\overline{T Q}$ is a compact convex set and $T$ maps $\overline{T Q}$ into itself. It follows that $T$ has a fixed point in $Q$; that is, there exists a function $p(x)$ satisfying (10.4) and

$$
\iint p(g x) \sigma_{\phi}(g, x) \psi(g) d g=\lambda p(x) .
$$

If we set $f_{x}(g)=p(g x) \sigma_{\phi}(g, x)$, we then find that $f_{x} \in V_{\lambda}(\psi)$. Now

$$
\int f_{x}(g) d \theta_{\xi}(x)=\int p(g x) \sigma_{\phi}(g, x) d \theta_{\xi}(x)=\int p(x) \sigma_{\phi}\left(g, g^{-1} x\right) d g \theta_{\xi}(x) .
$$

By (10.2), this gives

$$
\int f_{x}(g) d \theta_{\xi}(x)=\int p(x) \sigma^{\prime}(g, \xi) d \theta_{o \xi}(x)=\sigma^{\prime}(g, \xi) p^{\prime}(g \xi)=p^{\prime}(\xi) s^{\prime}(g, \xi),
$$

since $p \in Q$. This expresses $s^{\prime}(\cdot, \xi)$ as a linear combination of the functions $f_{x}$ which are in $V_{\lambda}(\psi)$. However, the $s^{\prime}(\cdot, \xi)$ were taken to be extremals of $V_{\lambda}(\psi)$. It must therefore be the case that all the $f_{x}$ with $x$ in the support of $\theta_{\xi}$ are proportional. If $j$ were not 1-1, that is, $j\left(x_{1}\right)=j\left(x_{2}\right)$ for some pair $x_{1}, x_{2} \in B(G)$, then, since $f_{x_{1}}$ and $f_{x_{2}}$ are proportional,

$$
\frac{p\left(g x_{1}\right)}{p\left(x_{1}\right)} \sigma_{\phi}\left(g, x_{1}\right)=\frac{p\left(g x_{2}\right)}{p\left(x_{2}\right)} \sigma_{\phi}\left(g, x_{2}\right) .
$$


Replace $g$ by $k g$ and integrate both sides of (10.8) over $K$. This gives

$$
\frac{\sigma_{\phi}\left(g, x_{1}\right)}{p\left(x_{1}\right)}=\frac{\sigma_{\phi}\left(g, x_{2}\right)}{p\left(x_{2}\right)} .
$$

Setting $g=e$ we find $p\left(x_{1}\right)=p\left(x_{2}\right)$ and $\sigma_{\phi}\left(\cdot, x_{1}\right)=\sigma_{\phi}\left(\cdot, x_{2}\right)$. Since $\sigma_{\phi}$ is basic this cannot happen unless $x_{1}=x_{2}$. This proves that $j$ is $1-1$ and hence that $B^{\prime}=B(G)$. This completes the proof of the theorem.

As a result, the statement of Theorem 8.1 may be made more precise. That is, the normalized extremals of $V_{\lambda}(\psi)$, which are already known to be multiplier functions, are now known to be basic multiplier functions. We now give a more precise form to the Choquet-Deny representation for $V_{\lambda}(\psi)$. For each cohomology class $\eta \in H(B(G)$ ), we denote by $\sigma_{\eta}$ the unique $K$-multiplier in that class. $\sigma_{\eta}$ belongs to a spherical function which we shall denote $\phi_{\eta}$.

Lemma 10.1. For each cohomology class $\eta \in H(B(G))$, there exists a uniquely determined positive constant $\Lambda(\eta)$ and a positive continuous function $p_{\eta}(x)$ on $B(G)$, unique up to a constant multiple, such that

$$
\int p_{\eta}(g x) \sigma_{\eta}(g, x) \psi(g) d g=\Lambda(\eta) p_{\eta}(x) .
$$

Proof. Let $Q$ denote the convex set of non-negative functions $p(x)$ on $B(G)$ satisfying $\int p(x) d m(x)=1$. Let $T$ denote the transformation of $Q$ into itself defined by

$$
T p(x)=c(p) \int p(g x) \sigma_{\eta}(g, x) \psi(g) d g,
$$

where $c(p)$ is a constant chosen so that $T p \in Q$. One verifies that the functions of $T Q$ form an equicontinuous family so that $\overline{T Q}$ is a compact convex set. If $p_{\eta}$ is the fixed point of $T$ in $\overline{T Q}$ then there is a constant $\Lambda(\eta)$ so that (10.9) is valid.

The uniqueness of $\Lambda(\eta)$ follows from

$$
\frac{\int p(g x) \sigma_{\eta}(g, x) \psi(g) d g}{\int p^{\prime}(g x) \sigma_{\eta}(g, x) \psi(g) d g}=\frac{\lambda}{\lambda^{\prime}} \frac{p(x)}{p^{\prime}(x)} .
$$

If $\lambda / \lambda^{\prime}<1$ we choose $x$ so that $p / p^{\prime}$ attains its minimum at $x$; then (10.10) becomes contradictory. So $\lambda \geqq \lambda^{\prime}$ and similarly $\lambda^{\prime} \leqq \lambda$. Thus there is a unique eigenvalue for which (10.9) can have a solution. Now if $p_{\eta}$ satisfies (10.9), then the multiplier functions 


$$
\frac{p_{\eta}(g x)}{p_{\eta}(x)} \sigma_{\eta}(g, x)
$$

belong to $V_{\Delta(\eta)}(\psi)$, and, by Lemma $8.1, p_{\eta}$ is thereby uniquely determined. This completes the proof of the lemma.

If we compare the multiplier functions on $G$ with the exponentials $e^{a t}$ on the real line, then we see that $\Lambda(\eta)$ corresponds to the Laplace transform of the functions $\psi(g)$.

We can now formulate the Choquet-Deny representation as follows:

THEOREM 10.2. Restrict the function $\Lambda(\eta)$ to the subset $H_{b}(B(G)$ ) $\subset H(B(G))$, so that for each $\lambda>0, \Lambda^{-1}(\lambda) \subset H_{b}(B(G))$. Then, for each function $f \in V_{\lambda}(\psi)$, there exists a measure $\omega$ on $\Lambda^{-1}(\lambda) \times B(G)$ such that

$$
f(g)=\int_{\Lambda^{-1}(\lambda) \times B(\theta)} p_{\eta}(g x) \sigma_{\eta}(g, x) d \omega(\eta, x) .
$$

In considering $\Lambda^{-1}(\lambda) \times B(G)$ as a measure space, we have imbedded it in $V_{\lambda}(\psi)$ and taken the induced Borel structure. We do not as yet know enough about the function $\Lambda(\eta)$ to claim that $\Lambda^{-1}(\lambda)$ is a Borel subset of $H(B(G))$. tion

What has been gained by our analysis as regards the integral equa-

$$
\int_{G} f\left(g^{\prime} g\right) \psi\left(g^{\prime}\right) d g^{\prime}=\lambda f(g)
$$

is this. We have replaced a single equation for a function on a noncompact space and which, moreover, does not have a unique solution, by a family of equations (10.9) for a function $\Lambda(\eta)$ and a family of functions $p_{\eta}(x)$ on the compact space $B(G)$ which now have a unique solutions.

Theorem 10.2 still leaves open the uniqueness of the measure $\omega$. Although we know that the representation in terms of normalized extremals of $V_{\lambda}(\psi)$ is unique, we have not shown that every multiplier function corresponding to a point in $\Lambda^{-1}(\lambda) \times B(G)$ is an extremal. This we shall do in the next theorem.

THEOREM 10.3. The normalized extremals of $V_{\lambda}(\psi)$ consist exactly of those multiplier functions $s(\cdot, x), x \in B(G)$, such that $s$ is basic, and $s(\cdot, x)$ belongs to $V_{\lambda}(\psi)$.

Proof. We have already shown in Theorem 10.1 that if $s(\cdot, x)$ is extremal in $V_{\lambda}(\psi)$ then $s$ is basic. We now show the converse. Suppose 
then that $s$ is basic and $s(\cdot, x) \in V_{\lambda}(\psi)$. By Theorem 10.2 there is some measure $\omega$ with

$$
s\left(g, x_{0}\right)=\int_{\mathbb{A}^{-1}(\lambda) \times B(G)} p_{\eta}(g x) \sigma_{\eta}(g, x) d \omega(\eta, x) .
$$

Here we may assume that the multiplier functions

$$
s_{\eta}(g, x)=\frac{p_{\eta}(g x)}{p_{\eta}(x)} \sigma_{\eta}(g, x)
$$

are extremals of $V_{\lambda}(\psi)$ for those $\eta$ occurring in this representation. If $s \sim \sigma$ where $\sigma \in M_{K}(B(G))$, then, by (2.3),

$$
\int_{\boldsymbol{K}} s(k g, x) d k=\left(\int_{\boldsymbol{K}} s(k, x) d k\right) \sigma(g, x)=S(x) \sigma(g, x) .
$$

By (10.12)

$$
S\left(x_{0}\right) \sigma\left(g, x_{0}\right)=\int_{\mathbb{A}^{-1}(\lambda) \times B(G)}\left(\int p_{\eta}(y) d m(y)\right) \sigma_{\eta}(g, x) d \omega(\eta, x)
$$

or

$$
\sigma\left(g, x_{0}\right)=\int_{\Lambda^{-1}(\lambda) \times B(G)} \sigma_{\eta}(g, x) d \omega^{\prime}(\eta, x) .
$$

Setting $g=e$, we see that $\omega^{\prime}$ is a probability measure. Replacing $g$ by $g k$ and integrating both sides of (10.14) over $K$ we find

$$
\phi(g)=\int_{\Lambda^{-1}(\lambda)} \phi_{\eta}(g) d \omega^{\prime \prime}(\eta) .
$$

Here $\phi$ is the spherical function to which $\sigma$ belongs. Now replace $g$ by $g k g$ and integrate over $K$. We have, by (3.2),

$$
\begin{aligned}
\phi(g)^{2} & =\int_{\Delta^{-1}(\lambda)} \phi_{\eta}(g)^{2} d \omega^{\prime \prime}(\eta) \\
& =\left\{\int_{\Delta^{-1}(\lambda)} \phi_{\eta}(g) d \omega^{\prime \prime}(\eta)\right\}^{2} .
\end{aligned}
$$

Now $\omega^{\prime \prime}$ is again a probability measure, so (10.15) shows that $\phi_{\eta}$ is essentially constant as a function of $\eta$. This means that $\omega^{\prime \prime}$ is concentrated on classes $\eta$ with the property that $\phi_{\eta}=\phi$. All we need, however, is the fact that for some $\eta, \phi_{\eta}=\phi$. We have $s$ basic and $\sigma \sim s$. It follows that $\sigma$ is basic; so $\sigma=\sigma_{\phi^{\prime}}$ for some spherical function $\phi^{\prime}$, by 
Theorem 9.1. Since $\sigma_{\phi^{\prime}}$ belongs to $\phi^{\prime}$ and $\sigma$ belongs to $\phi$, we must have $\phi^{\prime}=\phi$ and so $\sigma=\sigma_{\phi}$. Since the $\eta$ occurring in (10.12) are basic classes, $\sigma_{\eta}$ is basic and so $\sigma_{\eta}=\sigma_{\phi_{\eta}}=\sigma_{\phi}=\sigma$. As a result, $s \sim \sigma_{\eta}$. But $\sigma_{\eta} \sim s_{\eta}$ $((10.13))$, so $s \sim s_{\eta}$. Now the multiplier functions $s(\cdot, x)$ and $s_{\eta}(\cdot, x)$ are in $V_{\lambda}(\psi)$ and, by Lemma 8.1, since $s \sim s_{\eta}$, we must have $s=s_{\eta}$. Since the functions $s_{\eta}(\cdot, x)$ are extremal, it follows that the functions $s(\cdot, x)$ are extremal and this proves our theorem.

This theorem implies that the normalized extremals of $V_{\lambda}(\psi)$ correspond exactly to the points of $\Lambda^{-1}(\lambda) \times B(G)$, the correspondence being given by

$$
(\eta, x) \rightarrow \frac{p_{\eta}(g x)}{p_{\eta}(x)} \sigma_{\eta}(g, x) .
$$

Since the representation of elements in $V_{\lambda}(\psi)$ in terms of measures on the normalized extremals is unique, we have:

Corollary. For each function $f \in V_{\lambda}(\psi)$, the measure $\omega$ in (10.11) is unique.

The eigenvalue function $\Lambda(\eta)$ was defined originally for all $\eta \in H(B(G))$. It is not an arbitrary function; in particular, it is determined in all of $H(B(G))$ by its values in $H_{b}(B(G))$. This follows from the fact that the Choquet-Deny representation depends only on the restriction of $\Lambda(\eta)$ to $H_{b}(B(G))$. However, we can make a more precise statement.

Definition 10.1. We shall say that two classes $\eta_{1}$ and $\eta_{2}$ in $H(B(G)$ ) are related if $\phi_{\eta_{1}}=\phi_{\eta_{2}}$.

Thus every class is related to one and only one basic class.

Theorem 10.4. If $\eta_{1}$ and $\eta_{2}$ are related, then $\Lambda\left(\eta_{1}\right)=\Lambda\left(\eta_{2}\right)$.

Proof. Suppose $s$ is a multiplier in the class $\eta_{1}$ such that $s(\cdot, x)$ $\in V_{\lambda}(\psi)$. Then $\lambda=\Lambda\left(\eta_{1}\right)$. By Theorem 10.2,

$$
s\left(g, x_{0}\right)=\int_{\Lambda^{-1}(\lambda) \times B(G)} p_{\eta}(g x) \sigma_{\eta}(g, x) d \omega(\eta, x) .
$$

Replacing $g$ by $k_{1} g k_{2}$ and integrating both sides of (10.16) over $K \times K$ we obtain, as in the proof of Theorem 10.3,

$$
\phi_{\eta_{1}}(g)=\int_{\Lambda^{-1}(\lambda)} \phi_{\eta}(g) d \omega^{\prime \prime}(\eta) .
$$

As in the proof of Theorem 10.3, it follows that $\eta$ is constant in the 
integration (10.17), and $\phi_{\eta_{1}}=\phi_{\eta}$. Since $\eta \in \Lambda^{-1}(\lambda)$, we have $\lambda=\Lambda\left(\eta_{1}\right)$ $=\Lambda(\eta)$. Now $\eta$ is the uniquely determined basic class related to $\eta_{1}$ since $\phi_{\eta_{1}}=\phi_{\eta}$ and $\eta$ is basic. The same reasoning then implies $\Lambda\left(\eta_{2}\right)$ $=\Lambda(\eta)$, so $\Lambda\left(\eta_{1}\right)=\Lambda\left(\eta_{2}\right)$. This completes the proof of the theorem.

11. Irreducible multipliers. If $s \in M(B(G))$, let $V(s)$ denote the closed cone of functions on $G$ generated by $s(\cdot, x), x \in B(G)$. Here closure will mean with respect to the topology of pointwise convergence on $G . V(s)$ is identical with the functions

$$
f_{\omega}(g)=\int_{B(G)} s(g, x) d \omega(x)
$$

where $\omega$ ranges over the positive measures on $B(G)$. That this family is closed follows from two facts. First, if $f_{\omega}(e)$ is bounded, then the measures $\omega$ are bounded and lie in a compact set. Secondly, the map $\omega \rightarrow f_{\omega}$ is continuous with respect to the weak topology on positive measures.

The cones $V(s)$ are obviously closed under (right) translation. We may inquire for which $s$ the cone $V(s)$ will be irreducible, that is, have no proper closed, translation-invariant subcone. For example, if $\nu$ is a smooth measure on $B(G)$, the multiplier $s(g, x)=\left(d g^{-1} \nu / d \nu\right)(x)$ is not irreducible. For $\int s(g, x) d \nu(x)=\nu(B(G))$ is constant, and if $V(s)$ contains a constant it cannot be irreducible unless it reduces to the cone of positive constants.

Definition 11.1. We say $s \in M(B(G))$ is irreducible if the cone $V(s)$ contains no proper closed translation-invariant subcone.

Let $m$ denote the $K$-invariant probability measure on $B(G)$. We shall use $\sigma_{0}$ to denote the $K$-multiplier defined by

$$
\sigma_{0}(g, x)=\frac{d g^{-1} m}{d m}(x)
$$

LEMMA 11.1. If $s^{*}$ is a basic multiplier, then $s=\sigma_{0} / s^{*}$ is irreducible.

Proof. A typical function in $V(s)$ is $f(g)=\int s(g, x) d \omega(x)$. To prove that $s$ is irreducible, we must show that given any $f \in V(s)$, the functions $s(\cdot, x)$ can be retrieved from $f$ by taking limits of positive linear combinations of translates. We first form

$$
F(g)=\int_{K} f(g k) d k=\int_{K} \int_{B(G)} s(g, k x) s(k, x) d \omega(x) d k .
$$

Now, fixing $x_{0} \in B(G)$, let $x=k^{\prime} x_{0}$. Then 


$$
\begin{aligned}
\int_{K} s(g, k x) s(k, x) d k & =\frac{1}{s\left(k^{\prime}, x_{0}\right)} \int_{K} s\left(g, k k^{\prime} x_{0}\right) s\left(k k^{\prime}, x_{0}\right) d k \\
& =\frac{1}{s\left(k^{\prime}, x_{0}\right)} \int_{K} s\left(g, k x_{0}\right) s\left(k, x_{0}\right) d k=\frac{1}{s\left(k^{\prime}, x_{0}\right)} F_{0}(g) .
\end{aligned}
$$

From (11.2) and (11.3) we see that $F(g)$ is proportional to $F_{0}(g)$, where the latter function is independent of $\omega$, and thus independent of the function $f$ in $V(s)$. It follows that the function $F_{0}(g)$ belongs to every closed translation-invariant subcone of $V(s) . F_{0}(g)$ may be rewritten as

$$
F_{0}(g)=\int_{B(G)} s(g, x) h(x) d m(x),
$$

where $h(x)$ is a positive continuous function on $B(G)$.

Recall Lemma 9.3 according to which $s^{*}$ is basic only if the functions of the form $\int s^{*}(g, x) d \mu(g), \mu \geqq 0$, are dense in the positive continuous functions on $B(G)$. In particular, if $U$ is any neighborhood of a point $x_{0} \in B(G)$ and $\epsilon>0$, there exists a measure $\mu$ on $G$ with

$$
N(x)=\int s^{*}(g, x) d \mu(g)<\epsilon \text { for } x \notin U,
$$

$$
\int_{B(G)} N(x) d m(x)=1
$$

Then

$$
\begin{aligned}
F_{0}\left(g \gamma^{-1}\right) & =\int_{B(G)} s\left(g, \gamma^{-1} x\right) s\left(\gamma^{-1}, x\right) h(x) d m(x) \\
& =\int_{B(G)} s(g, x) s\left(\gamma^{-1}, \gamma x\right) h(\gamma x) d \gamma^{-1} m(x) \\
& =\int_{B(G)} s(g, x) s(\gamma, x)^{-1} h(\gamma x) \sigma_{0}(\gamma, x) d m(x) \\
& =\int_{B(G)} s(g, x) s^{*}(\gamma, x) h(\gamma x) d m(x) .
\end{aligned}
$$

So

$$
\int_{G} F_{0}\left(g \gamma^{-1}\right) d \mu(\gamma)=\int_{B(G)} s(g, x)\left[\int_{G} s^{*}(\gamma, x) h(\gamma x) d \mu(\gamma)\right] d m(x) .
$$


Since $\int_{G} s^{*}(\gamma, x) d \mu(\gamma)$ approximates a $\delta$-function in $x$ with respect to the measure $m$, and since $h(x)$ is bounded from above and below, it follows that

$$
\int_{G} s^{*}(\gamma, x) h(\gamma x) d \mu(\gamma)
$$

approximates a multiple of the same $\delta$-function. More precisely, we see from (11.5) that

$$
\left|\frac{\int F_{0}\left(g \gamma^{-1}\right) d \mu(\gamma)}{\int F_{0}\left(\gamma^{-1}\right) d \mu(\gamma)}-s\left(g, x_{0}\right)\right| \leqq \max _{x \in U} C\left|s(g, x)-s\left(g, x_{0}\right)\right|,
$$

where $C$ is a constant depending upon $h(x)$. Consequently, with an appropriate choice of measures $\mu_{n}$,

$$
\frac{\int F_{0}\left(g \gamma^{-1}\right) d \mu_{n}(\gamma)}{\int F_{0}\left(\gamma^{-1}\right) d \mu_{n}(\gamma)} \rightarrow s\left(g, x_{0}\right), \text { as } n \rightarrow \infty,
$$

for each $g$. It follows that $s\left(\cdot, x_{0}\right)$ belongs to every closed translationinvariant cone containing $F_{0}(g)$ and so $V(s)$ is irreducible. This completes the proof of the lemma.

We turn now to $K$-multipliers.

Lemma 11.2. For each spherical function $\phi$ there is one and only one $K$-multiplier belonging to $\phi$ which is irreducible.

Proof. That at least one $K$-multiplier belonging to $\phi$ is irreducible is easily established. If $\phi(g)$ is spherical, then $\tilde{\phi}(g)=\phi\left(g^{-1}\right)$ is also spherical. Suppose $\sigma^{*}$ is the basic multiplier belonging to $\tilde{\phi}$, and form $\sigma=\sigma_{0} / \sigma^{*}$. Then

$$
\begin{aligned}
\int_{B(G)} \sigma(g, x) d m(x) & =\int_{B(G)} \sigma\left(g, g^{-1} x\right) \frac{d g m}{d m}(x) d m(x) \\
& =\int_{B(G)} \frac{1}{\sigma\left(g^{-1}, x\right)} \sigma_{0}\left(g^{-1}, x\right) d m(x) \\
& =\int_{B(G)} \sigma^{*}\left(g^{-1}, x\right) d m(x) \\
& =\delta\left(g^{-1}\right)=\phi(g) .
\end{aligned}
$$


So $\sigma$ belongs to $\phi$ and is irreducible by the foregoing lemma.

Now suppose that $\sigma_{1}$ and $\sigma_{2}$ are related $K$-multipliers (Definition 10.1) which are both irreducible. Since $\phi(g)=\int_{B(G)} \sigma_{i}(g, x) d m(x)$, we have $\phi \in V\left(\sigma_{1}\right) \cap V\left(\sigma_{2}\right)$. If both cones are irreducible, we must have $V\left(\sigma_{1}\right)=V\left(\sigma_{2}\right)$. In particular, we have

$$
\begin{aligned}
& \sigma_{2}\left(g, x_{0}\right)=\int \sigma_{1}(g, x) d \omega_{1}(x), \\
& \sigma_{1}\left(g, x_{0}\right)=\int \sigma_{2}(g, x) d \omega_{2}(x)
\end{aligned}
$$

for two positive measures $\omega_{1}, \omega_{2}$ on $B(G)$. One can find measures $\mu_{1}, \mu_{2} \geqq 0$ on $K$ with $\omega_{i}=\mu_{i} * x_{0}$, and (11.6) becomes

$$
\begin{aligned}
& \sigma_{2}\left(g, x_{0}\right)=\int_{K} \sigma_{1}\left(g k, x_{0}\right) d \mu_{1}(k), \\
& \sigma_{1}\left(g, x_{0}\right)=\int_{K} \sigma_{2}\left(g k, x_{0}\right) d \mu_{2}(k) .
\end{aligned}
$$

Combining these two:

$$
\sigma_{1}\left(g, x_{0}\right)=\int_{K} \sigma_{1}\left(g k, x_{0}\right) d \mu_{2} * \mu_{1}(k) .
$$

Let $\mu^{(n)}$ denote the $n$-fold convolution of $\mu_{2} * \mu_{1}$ with itself. It is not hard to see that $n^{-1}\left[\mu^{(1)}+\cdots+\mu^{(n)}\right]$ converges, as $n \rightarrow \infty$, to the Haar measure of the least subgroup $K_{1} \subset K$ which contains the support of $\mu_{2} * \mu_{1}\left[6\right.$, pp. 343-344]. Since (11.7) would be valid with $\mu^{(n)}$ replacing $\mu_{2} * \mu_{1}$, it follows that one may write

$$
\sigma_{1}\left(g, x_{0}\right)=\int_{K_{1}} \sigma_{1}\left(g k, x_{0}\right) d k .
$$

Hence, $\sigma_{1}\left(g k, x_{0}\right)=\sigma_{1}\left(g, x_{0}\right)$ for every $k \in K_{1}$. If $P_{2}$ and $P_{1}$ denote, respectively, the supports of $\mu_{2}$ and $\mu_{1}$, then $P_{2} P_{1} \subset K_{1}$. In particular, $P_{1}$ is contained in some left coset $k_{1} K_{1}$. Then

$$
\sigma_{2}\left(g, x_{0}\right)=\int_{K_{1}} \sigma_{1}\left(g k_{1} k, x_{0}\right) d k_{1}^{-1} \mu_{1}(k) .
$$

But the support of $k_{1}^{-1} \mu_{1}$ is in $K_{1}$ and the $k \in K_{1}$ leave $\sigma_{1}$ invariant. So

$$
\sigma_{2}\left(g, x_{0}\right)=\sigma_{1}\left(g k_{1}, x_{0}\right)=\sigma_{1}\left(g, k_{1} x_{0}\right)=\sigma_{1}\left(g, x_{1}\right) .
$$

But we have seen in $\$ 7$, using Lemma 7.2 , that this implies $\sigma_{1}=\sigma_{2}$. 
This completes the proof of the lemma.

We can now prove the main result of this section.

THEOREM 11.1. A multiplier $s$ is irreducible if and only if $\sigma_{0} / s$ is basic.

Proof. The implication in one direction is given in Lemma 11.1. What remains to be shown is that if $s$ is irreducible, then $\sigma_{0} / s$ is basic. We claim that if $s$ is irreducible and $\sigma$ is the $K$-multiplier equivalent to $s$, then $\sigma$ is irreducible. To see this, consider the operator with domain $V(s)$ defined by

$$
T f(g)=\int_{K} f(k g) d k=m_{K} * f(g) .
$$

Since $T$ commutes with right translation, it follows that $T V(s)$ is a translation-invariant cone. Moreover,

$$
T s(g, x)=\int_{K} s(k g, x) d k=\sigma(g, x)\left[\int s(k, x) d k\right]
$$

so that $\sigma(\cdot, x) \in T V(s)$. It is also easy to see that $T V(s)$ is closed, so it follows that $T V(s) \supset V(\sigma)$. Now, since $V(s)$ is irreducible, it follows that $T V(s)$ is irreducible, which implies that $T V(s)=V(\sigma)$, and that $V(\sigma)$ is irreducible, whence $\sigma$ is irreducible. Let $\sigma$ belong to $\phi$. By the foregoing lemma, $\sigma$ is the only irreducible multiplier belonging to $\phi$. On the other hand, we know that if $\sigma^{*}$ is the basic multiplier belonging to $\phi\left(g^{-1}\right)$, then $\sigma_{0} / \sigma^{*}$ is irreducible and belongs to $\phi(g)$. So we must have $\sigma=\sigma_{0} / \sigma^{*}$. In other words, if $\sigma$ is irreducible, $\sigma_{0} / \sigma$ is basic. But then $\sigma_{0} / s$ is basic, for $\sigma \sim s$ implies $\sigma_{0} / \sigma \sim \sigma_{0} / s$. This completes the proof of the theorem.

CoRollary. If $s_{1}$ and $s_{2}$ are equivalent multipliers in $M(B(G))$, then $s_{1}$ is irreducible if and only if $s_{2}$ is irreducible.

If we consider the exponentials in the real line we notice that they obviously have the irreducibility property-the translation-invariant cones they generate are irreducible (in fact, 1-dimensional). Thus, for the real line, the properties of irreducibility and forming the extremals in the Choquet-Deny representation are shared by the same class of functions. In the case of semi-simple groups these properties split: one characterizes the irreducible multiplier functions and the other the basic multiplier functions. (It is probably true that the only multipliers which are simultaneously basic and irreducible are those equivalent to $\sigma_{0}^{1 / 2}$.) 
12. Irreducible cones. We have mentioned the fact that the positive exponentials on the group of reals generate irreducible translation-invariant cones. In fact this property characterizes the exponentials. More precisely

THEOREM 12.1. If $V$ is a cone of non-negative continuous functions on the reals, closed under translation and closed in the topology of pointwise convergence, and if $V$ is irreducible (that is, no proper subcone of $V$ has the same properties), then $V$ consists of the positive multiples of some exponential $f(t)=e^{a t},-\infty<a<\infty$.

We will not give the proof of this inasmuch as it resembles that of the analogous result for semi-simple groups which we will give. Let us, however, sketch an alternative proof. If we know that $V$ has a compact base, then, since the reals form a Tychonoff group and act on $V$ by translation, $V$ must have a fixed ray, i.e., a 1-dimensional translation-invariant subcone. This must be all of $V$ and, as is easily seen, this implies that $V$ consists of multiples of an exponential. The problem is, therefore, to show that $V$ has a compact base. This may be done by showing that if $V$ is irreducible, the functions in $V$ are convex, secondly, that, in fact, their logarithms are convex, and, finally, that their logarithms satisfy a uniform Lipschitz condition.

For an arbitrary topological group one can attempt to characterize the irreducible (right) translation-invariant cones. In the semi-simple case, this is most easily done if we assume further that the functions in the cone are invariant under left translation by elements in a maximal compact subgroup. Note that if a cone is irreducible (with respect to right translations) and if one function has this property, then so do all functions in the cone.

TheOREM 12.2. Let $V$ be a cone of continuous non-negative functions on $G$ closed under (right) translation and closed in the topology of pointwise convergence. Assume $V$ is irreducible, that is, that no proper subcone of $V$ has these properties. If, furthermore, there is a function $f \in V$ with $f(k g)=f(g)$ for all $k \in K$, then $V$ coincides with $V(\sigma)$ for some irreducible $K$-multiplier $\sigma$.

We remark that since the functions of $V$ may be thought of as functions on $K \backslash G$, the question is that of characterizing cones of nonnegative functions on a symmetric space, invariant under the isometries of the space, and irreducible with respect to this property.

Proof. We convert the problem into one involving cones of measures. Identify $V$ with a subcone of the cone of positive (unbounded) measures on $G$ and let $\tilde{V}$ denote the closure of $V$ in this space. Sup- 
pose $\omega$ is a measure on $G$ corresponding to a continuous function of compact support. Then $V * \omega \subset V$ by the translation invariance of $V$, and, since $\pi_{n} \rightarrow \pi$ weakly implies $\pi_{n} * \omega \rightarrow \pi * \omega$ pointwise as functions, it follows that $\tilde{V} * \omega \subset V$. Suppose that $\tilde{V}$ is not irreducible, and suppose that $W \subset \tilde{V}$ is also invariant and weakly closed. Then $W * \omega \subset W \cap V$ so, then, $W \cap V$ is nonempty. By the irreducibility of $V, W \cap V=V$ or $W \supset V$ and, since $W$ is weakly closed, $W=\tilde{V}$. Hence $\tilde{V}$ is irreducible.

Let $\tilde{V}_{K}$ denote the set of measures $\pi \in \tilde{V}$ such that $\pi * k=\pi$ for each $k \in K$. Under the hypotheses of the theorem, it is true, for every $\pi \in V$, that $k * \pi=\pi$. So the measures in $V_{K}$ have the form $m_{K} * \pi^{\prime} * m_{K}$. By Lemma 7.1, measures of this form are commutative when the product is defined.

Notice that $V_{K}$ is invariant under the operations $\pi \rightarrow \pi * \gamma * m_{K}$, $\gamma \in G$. We claim that $V_{K}$ is minimal with respect to this property. Let $W \subset \tilde{V}_{K}$ be closed and invariant under $\pi \rightarrow \pi * \gamma * m_{K}$ for all $\gamma \in G$. Form $W^{\prime}=\left\{\pi \in \tilde{V}: \pi * g * m_{K} \in W\right.$ for all $\left.g \in G\right\}$. Clearly $W^{\prime}$ is rightinvariant, weakly closed, and $W^{\prime} \supset W$ so it is not empty. So $W^{\prime}=\tilde{V}$, or $\tilde{V} * m_{K}=\widetilde{V}_{K} \subset W$. Thus $W=V_{K}$.

Let $\omega$ be a measure of compact support on $G$ and form $\tilde{V}_{K} * \omega * m_{K}$. This cone is closed, for, if $\pi_{n} * \omega * m_{K}$ converges, a subsequence of $\left\{\pi_{n}\right\}$ must converge weakly $\left(\pi * \omega * m_{K}\left(\Delta_{1} \Delta_{2}\right) \geqq \pi\left(\Delta_{1}\right) \omega * m_{K}\left(\Delta_{2}\right)\right)$. We also have $\tilde{V}_{K} * \omega * m_{K} * \gamma * m_{K}=\tilde{V}_{K} * \gamma * m_{K} * \omega * m_{K} \subset \tilde{V}_{K} * \omega * m_{K}$. Also $\tilde{V}_{K} * \omega * m_{K} \subset \tilde{V} * m_{K}=\tilde{V}_{K}$, so that $\tilde{V}_{K} * \omega * m_{K}$ is a subcone of $V_{K}$ invariant under $\pi \rightarrow \pi * \gamma * m_{K}$. So $\tilde{V}_{K} * \omega * m_{K}=\tilde{V}_{K}$.

Now $V_{K}$ is a weakly closed cone of positive measures on $G$, so, by Theorem 6.1, it is spanned by its extremals. Let $\pi_{0}$ be an extremal of $\tilde{V}_{K}$. By what has just been shown $\pi_{0}=\pi_{\omega} * \omega * m_{K}$ for some measure $\pi_{\omega} \in \tilde{V}_{K}$. But this expresses $\pi_{0}$ as a linear combination of measures $\pi_{\omega} * g * m_{K} \in \widetilde{V}_{K}$. Since $\pi_{0}$ is extremal, these must be proportional for almost all $g$ in the support of $\omega$. By continuity, this will be true for all $g$ in the support of $\omega$. Moreover, if the identity of $G$ is in the support of $\omega$, then $\pi_{0}$ will itself be proportional to $\pi_{\omega}$. We then find that $\pi_{0} * g * m_{K}$ is proportional to $\pi_{0}$ for all $g$ in the support of $\omega$. Since $\omega$ had arbitrarily large support, we must have

$$
\pi_{0} * g^{-1} * m_{K}=\phi(g) \pi_{0} .
$$

Since $\pi_{0}=m_{K} * \pi_{0} * m_{K}$, we have

$\pi_{0} * g * m_{K}=m_{K} * \pi_{0} * m_{K} * g * m_{K}=m_{K} * g * m_{K} * \pi_{0} * m_{K}=m_{K} * g * \pi_{0}$,

so that 


$$
m_{K} * g^{-1} * \pi_{0}=\phi(g) \pi_{0} .
$$

This, however, is the defining equation (7.1) for semi-spherical functions. That is, $\phi$ must be spherical and $\pi_{0}$ corresponds to a function $\Phi \in V_{\phi}$. So

$$
\Phi(g)=\int_{B(G)} \sigma_{\phi}(g, x) d \nu(x)
$$

for some $\nu \geqq 0$ on $B(G)$. But $\Phi(g k)=\Phi(g)$ since $\pi_{0} \in \widetilde{V}_{k}$, so that

$$
\begin{aligned}
\Phi(g) & =\int_{B(G)} \int_{K} \sigma_{\phi}(g, k x) d k d \nu(x) \\
& =\int_{B(G)} \sigma_{\phi}(g, x) d m(x)=\phi(g) .
\end{aligned}
$$

We have proven that if $V$ is irreducible, some spherical function $\phi \in \tilde{V}$. Now $\tilde{V} * \omega \subset V$ if $\omega$ corresponds to a continuous function with compact support, and $\phi * \omega$ is proportional to $\phi$ if $\phi$ is spherical and $\omega$ is radial. Hence, $\phi \in V$. Now let $\sigma$ be the (unique) irreducible $K$ multiplier belonging to $\phi$ (Lemma 11.2). Then $V(\sigma)$ contains $\phi$ and, hence, $V(\sigma) \cap V$ is nonempty. It follows that $V=V(\sigma)$. This completes the proof of the theorem.

REMARK 1. The result would not have been altered if the topology on the cone $V$ had been taken to be that of uniform convergence on compact subsets.

REMARK 2. Whenever a group is represented by automorphisms of some structure, we may speak of an irreducible representation when the structure is minimal with respect to invariance under the action of the group. Traditionally, the structure is assumed to be that of a (topological) linear space; in this section we have taken it to be that of a closed cone. Another possibility would be to let the structure be that of a compact convex set. For example, every bounded measurable function on $G$ generates a translation-invariant compact convex set in $L^{\infty}(G)$ (with the weak* topology), and we may inquire when this gives an irreducible representation of $G$. Using [6] it is easy to show that, up to isomorphism, the irreducible representations of a semi-simple $G$ on compact convex sets are just its representations on the space of probability measures on one of its boundary spaces (the equivariant images of $B(G)$ ). With this it may be shown that the irreducible weak* compact convex sets in $L^{\infty}(G)$ are just those which are spanned by a function $f(g)$ satisfying $f(g k)=f(g)$ for all $k \in K$ and which, as a function on the symmetric space $G / K$, is harmonic. 
APPENDIX. LemMa 5.2. If $\pi$ is a measure on $G$ satisfying $t \pi=\chi(t) \pi$ for $t \in T$, and $\chi$ a positive character on $T$, then $\pi=m_{T}^{\chi} * \mu$ for some positive measure $\mu$ on $G$.

ProOF. Form the cone $V$ of measures $\pi$ on $G$ satisfying $t \pi=\chi(t) \pi$, $\pi \geqq 0$. Let $h(g)$ be a non-negative continuous function on $G$ with compact support and such that $h(k)>0$ for $k \in K$. If, for $\pi \in V, \pi(h)=0$, then $\int h(t g) d \pi(g)=0$ for all $t \in T$. But the interiors of the supports of the functions $h(\mathrm{tg})$ cover $G$ since $T K=G$. This would then imply that $\pi=0$, so we see that $\pi(h)>0$ for all $\pi \in V, \pi \neq 0$. We can now apply Theorem 6.1 to the weakly closed cone $V$, and we find that each $\pi \in V$ has an integral representation in terms of normalized extremals of $V$. We see then that the lemma will be proved once we establish that the extremals of $V$ are the measures $m_{T}^{\chi} * \gamma, \gamma \in G$, up to a multiplicative constant.

Let $\pi \in V$ and form the measure $\pi_{\Delta}$ given by

$$
\pi_{\Delta}(f)=\int f(g) C_{\Delta}(T g) d \pi(g)
$$

where $\Delta$ is a set in $T \backslash G$ and $C_{\Delta}$ denotes its characteristic function. Now it is readily seen that $\pi_{\Delta}$ again belongs to $V$. So if $\pi$ is extremal in $V$ it must be concentrated on a single coset $T \gamma$. Then $\pi * \gamma^{-1}=\pi_{0}$ is concentrated on $T$ and satisfies $t \pi_{0}=\chi(t) \pi_{0}$. Define the measure $\pi_{1}$ on $T$ by

$$
d \pi_{1}(t)=\chi(t) d \pi_{0}(t)
$$

If $\tau \in T$, then

$$
\frac{d \tau \pi_{1}}{d \tau \pi_{0}}(t)=\frac{d \pi_{1}}{d \pi_{0}}\left(\tau^{-1} t\right)=\chi\left(\tau^{-1} t\right) .
$$

But $\left(d \tau \pi_{0} / d \pi_{0}\right)(t)=\chi(\tau)$, so

$$
\frac{d \tau \pi_{1}}{d \pi_{0}}(t)=\frac{d \tau \pi_{1}}{d \tau \pi_{0}}(t) \frac{d \tau \pi_{0}}{d \pi_{0}}(t)=\chi\left(\tau^{-1} t\right) \chi(\tau)=\chi(t)=\frac{d \pi_{1}}{d \pi_{0}}(t) .
$$

Hence $\tau \pi_{1}=\pi_{1}$ for all $\tau \in T$. So $\pi_{1}$ is proportional to Haar measure on $T$ and

$$
d \pi_{0}(t)=c \chi\left(t^{-1}\right) d t
$$

and $\pi_{0}=c m_{T}^{x}$. Since every extremal $\pi$ of $V$ has the form $\pi_{0} * \gamma^{-1}$ for some $\gamma \in G$, our lemma is proven. 


\section{BIBLIOGRAPHY}

1. F. Bruhat, Sur les représentations induites des groupes de Lie, Bull. Soc. Math. France 84 (1956), 97-205.

2. G. Choquet, Le théorème de représentation intégrale dans les ensembles convexes compacts, Ann. Inst. Fourier (Grenoble) 10 (1960), 333-344.

3. G. Choquet et J. Deny, Sur l'équation de convolution $\mu=\mu * \sigma$, C. R. Acad. Sci. Paris 250 (1960), 799-801.

4. N. Dunford and J. T. Schwartz, Linear operators, Interscience, New York, 1958.

5. E. B. Dynkin, Non-negative eigenfunctions of the Laplace-Beltrami operator and Brownian motion on certain symmetric spaces, Dokl. Akad. Nauk SSSR 141 (1961), 288-291 (Russian); translated as Soviet Math. Dokl. 2 (1961), 1433-1436.

6. H. Furstenberg, A Poisson formula for semi-simple Lie groups, Ann. of Math. (2) 77 (1963), 335-386.

7. I. Gel'fand, Spherical functions on symmetric Riemann spaces, Dokl. Akad. Nauk SSSR 70 (1950), 5-8. (Russian)

8. S. Helgason, Differential geometry and symmetric spaces, Academic Press, New York, 1962.

9. L. Loomis, Unique direct integral decompositions on convex sets, Amer. J. Math. 84 (1962), 509-526.

10. C. C. Moore, Compactifications of symmetric spaces, Amer. J. Math. 86 (1964), 201-218.

11. Séminaire Sophus Lie, Théorie des algèbres de Lie, École Normale Supérieure 1954/1955.

UNIVERSITY OF MINNESOTA 